(C) 2018. This manuscript version is made available under the CC-BY-NC-ND 4.0 license http://creativecommons.org/ licenses/by-nc-nd/4.0/

\title{
Boundary Control of Transverse Motion of Flexible Marine Risers Under Stochastic Loads
}

\author{
K. D. Do \\ Department of Mechanical Engineering, Curtin University \\ Bentley, WA 6102, Australia
}

\begin{abstract}
A constructive design of boundary controllers is proposed to stabilize transverse motion of flexible marine risers under stochastic loads induced by restoring membrane and fluid/air velocity. For stability analysis and boundary control design, global well-posedness (existence and uniqueness) and global stability criteria are developed for nonlinear stochastic evolution systems in Hilbert space subject to both state-dependent and additive stochastic disturbances. These criteria are obtained by developing Lyapunov sufficient conditions from local well-posedness and stability results. The well-posedness and stability developments are not only applied to ensure well-posedness and almost sure (practical) stability of the variational (strong) solution of the stochastic riser system but also to other hyperbolic systems.
\end{abstract}

Keywords: Stochastic marine riser; Stochastic evolution system; Existence and uniqueness; Almost sure stability; Hilbert space.

\section{Introduction}

As offshore exploration and production of natural resources such as oil and gas enters ever-deeper waters, production risers transporting petroleum products from the sea-bed to a surface ship/rig become extremely slender (the ratio of average diameter to length can be of the order $10^{-5}$ ), and are subject to larger nonlinear vibration and deformation due to stochastic environmental loads, see Faltinsen (1993). Excessive vibration of the risers can cause premature fatigue and loops, and shut down of the system, see Sorensen (2005), which result in both devastating environmental and financial consequences.

A representative riser system is given in Fig. 1, where the riser is connected to the well at seabed and ship/rig via ball joints. Reducing vibration of risers can be carried out by active control systems and/or passive devices. Passive systems are well established and limited to shallow water due to drag, see Baek and Karniadakis (2009). This paper focuses on active control systems installed at the riser top-end. As such, this top-end is connected to an active heave compensation system, see Do and Pan (2008b). The horizontal motion is controlled by a dynamic positioning system actuated by the ship/rig thrusters, see Sorensen (2005), or a actuated guide tube mechanism.

In existing works, the riser is modeled by a distributed parameter system (DPS) governed by hyperbolic partial differential equations (PDEs). There are two typical approaches to the control design. In the modal control approach, a lumped parameter system (LPS) governed by

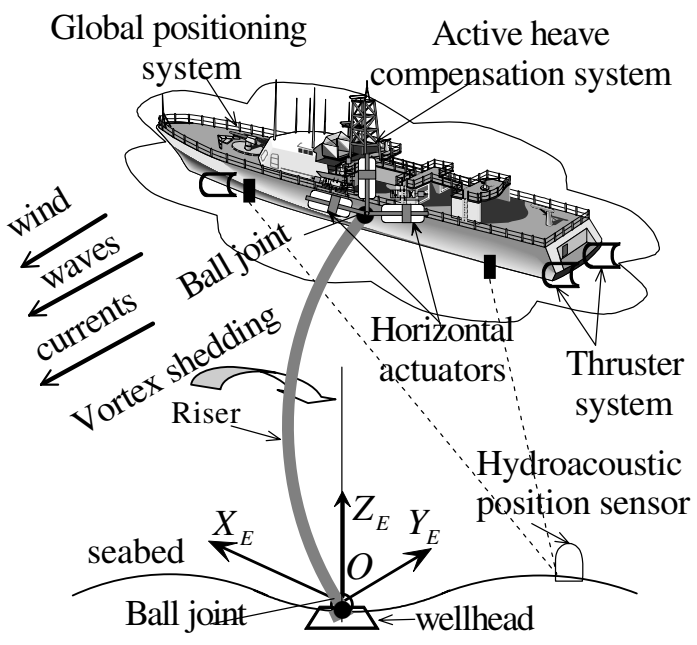
a set of ordinary differential equations (ODEs), described in terms of modal coordinates, is first obtained from the DPS by the Galerkin or assumed modes method. Then, classical control design methods, see Anderson and Moore

Email address: duc@curtin.edu . au (K. D. Do) 
(1990); Krstic et al. (1995); Khasminskii (1980); Deng et al. (2001); Mao (2007); Do (2015), can be applied to the LPS. This approach can only control a certain number of modes of a DPS and suffers from a spill-over problem, see Meirovitch (1997).

In the boundary control approach, the DPS is directly considered and controllers are implemented at the boundaries to control all the modes. Using the Lyapunov direct method in Khalil (2002), various boundary controllers of a proportional-derivative type have been proposed for flexible beam-like systems such as pipes and risers, see Stanway and Burrows (1981); Fard and Sagatun (2001); Fung et al. (1999); Fung and Tseng (1999); Do and Pan (2008a, 2009); Do (2011); Ge et al. (2010); He et al. (2011); Nguyen et al. (2013); Bohm et al. (2014); Do (2017, 2016). There is serious problem with all of the above works: the environmental loads on the riser were assumed to be deterministic. However, the loads induced by ocean currents, waves and wind are stochastic in nature Faltinsen (1993). This results in motion of the riser being described by a stochastic distributed-parameter system (SDPS) governed by stochastic partial differential equations (SPDEs). Well-posedness (existence and uniqueness) and stability of stochastic beams under and Lipschitz conditions subject to Dirichlet/Neumann boundary conditions (without boundary control) have been addressed by several mathematician, see Zhang (2007); Brzezniak et al. (2005); Chow and Menaldi (2014); Chow (2007). Various well-posedness and stability results developed for stochastic parabolic PDEs, see Pardoux (1979); Liu (2006); Prato and Zabczyk (1992); Liu and Mandrekar (1997); Gawarecki and Mandrekar (2011) and references therein are not directly applicable to analyze motion of the marine risers (flexible beams) under stochastic loads since motion of the risers are described by stochastic hyperbolic PDEs. This issue is mainly due to difficulty when checking coercivity and growth conditions.

The above discussion motivates the writing of this paper on a design of boundary controllers stabilizing transverse motion of stochastic flexible marine risers. The control design and stability analysis are based on Lyapunov direct method, which is developed in this paper, for stochastic evolution systems (SESs) in Hilbert space to guarantee almost sure (a.s.) global practical stability of the variational (strong in the stochastic sense but weak in the PDE) solution of the marine riser transverse motion system. Since, in addition to marine risers, various systems encountered in practice, such as elastic plates, panels, shells, strings, and membranes, see Dowell (1975), are hyperbolic systems subject to both state-dependent and additive stochastic disturbances. Global well-posedness (existence and uniqueness) and a.s. global stability criteria are developed for nonlinear SESs subject to both state-dependent and additive stochastic disturbances.

\section{Problem formulation}

\subsection{Transverse motion of stochastic risers}

Assume that the plane sections of the beam remain plane after deformation (i.e., warping is neglected); the riser is locally stiff (i.e., cross sections do not deform and Poisson effect is neglected); the beam material is homogeneous, isotropic and linearly elastic (i.e., it obeys Hookes's law); torsional and distributed moments induced by environmental disturbances are neglected; and that the beam deforms in one vertical plane, and its axial motion is ignored. Based on the Hamiltonian principle, the equations of motion of the riser with a configuration as shown in Fig. 2 where the earth-fixed coordinate system is $O Y Z$ and both of the riser ends are connected to the fixed base and the ship/rig via ball joints, see Do and Pan (2008a), can be derived as

$$
\begin{aligned}
& \varrho a u_{t t}(z, t)=-E I u_{z z z z}(z, t)+\left(P_{0}+\frac{3 E a}{2} u_{z}^{2}(z, t)\right) u_{z z}(z, t) \\
& +q\left(z, t, u(z, t)-\vartheta(z, t), u_{t}(z, t)-v(z, t)\right), \\
& -E I u_{z z z}(L, t)+P_{0} u_{z}(L, t)+\frac{E a}{2} u_{z}^{3}(L, t)=\varphi_{B}, \\
& u_{z z}(0, t)=0, u_{z z}(L, t)=0, u(0, t)=0, \\
& u\left(z, t_{0}\right)=u_{0}(z), u_{t}\left(z, t_{0}\right)=u_{1}(z),
\end{aligned}
$$

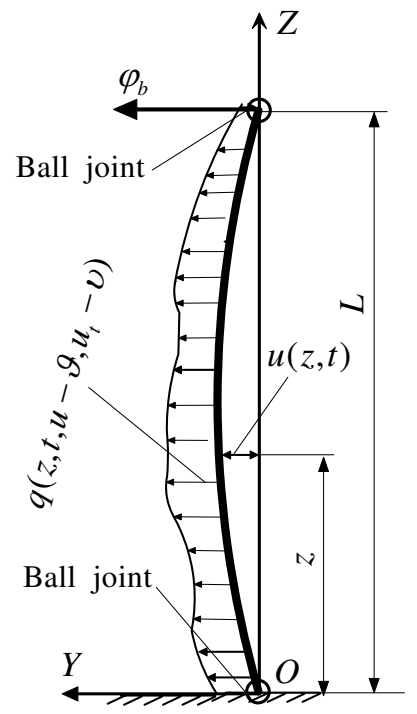

Figure 2: Riser coordinates.

where $\varrho$ is the mass density; $a$ is the cross section area of the riser; $u(z, t)$ denotes the transverse displacement of the riser at $(z, t) ; u_{t}(z, t)$ and $u_{z}(z, t)$ denote the partial derivatives of $u(z, t)$ with respect to time $t$ and the spatial coordinate $z$, and similarly for notations $u_{t t}(z, t), u_{z z}(z, t)$, and $u_{z z z z}(z, t) ; E$ is Young's modulus; $I$ is the moment of inertia of the riser cross section; $P_{0}$ is the constant axial force; $u_{0}(z)$ and $u_{1}(z)$ are initial values; $\varphi_{B}$ is the boundary 
control force; and $q(\bullet)$ with $\bullet$ being for $\left(z, t, u(z, t)-\vartheta(z, t), u_{t}(z, t)-v(z, t)\right)$ is the distributed force. This distributed force (nonlinearly) depends on the relative displacement $u(z, t)-\vartheta(z, t)$ between the riser and the membrane/spring (Elosta et al. (2013)), which is connected to the riser, and relative velocity $u_{t}(z, t)-v(z, t)$ between the riser and the fluid/air particle. In this paper, the distributed force $q(\bullet)$ is modelled by

$$
\begin{aligned}
q(\bullet)= & -c_{1}(z, t)(u(z, t)-\vartheta(z, t))-c_{2}(z, t)(u(z, t)-\vartheta(z, t))^{3} \\
& -d_{1}(z, t)\left(u_{t}(z, t)-v(z, t)\right)-d_{2}(z, t)\left(u_{t}(z, t)-v(z, t)\right)^{3},
\end{aligned}
$$

where $c_{1}(z, t)$ and $c_{2}(z, t)$ are referred to as the restoring coefficients while $d_{1}(z, t)$ and $d_{2}(z, t)$ are referred to as the damping coefficients. It is noted that the distributed force usually includes non-smooth restoring and damping terms such as $-c_{0}(z, t)|u(z, t)-\vartheta(z, t)|(u(z, t)-\vartheta(z, t))$ and $-d_{0}(z, t)\left|u_{t}(z, t)-v(z, t)\right|\left(u_{t}(z, t)-v(z, t)\right)$ with $c_{0}(z, t)$ and $d_{0}(z, t)$ being restoring and damping coefficients. However, these terms can be dominated by those terms already included in (2). Thus, we do not include the non-smooth terms in the distributed force $q(\bullet)$.

In general, the membrane displacement and air/fluid velocity consist of deterministic and stochastic parts, i.e., $\vartheta(z, t)$ and $v(z, t)$ can be written as

$$
\begin{aligned}
& \vartheta(z, t)=\vartheta_{d}(z, t)+\vartheta_{s}(z, t), \\
& v(z, t)=v_{d}(z, t)+v_{s}(z, t),
\end{aligned}
$$

where $\vartheta_{d}(z, t)$ and $\vartheta_{s}(z, t)$ are referred to as the deterministic and stochastic parts of $\vartheta(z, t)$, respectively; and similar notations for $v_{d}(z, t)$ and $v_{s}(z, t)$. Substituting (3) into (2) gives

$$
q(\bullet)=f\left(z, t, u(z, t), u_{t}(z, t)\right)+q_{s}\left(z, t, u(z, t), u_{t}(z, t), \vartheta_{s}(z, t), v_{s}(z, t)\right) .
$$

The deterministic part $f(\bullet)$ of $q(\bullet)$ is given by

$$
\begin{aligned}
f(\bullet)= & -a_{1}(z, t) u(z, t)+a_{2}(z, t) u^{2}(z, t)-a_{3}(z, t) u^{3}(z, t) \\
& -b_{1}(z, t) u_{t}(z, t)+b_{2}(z, t) u_{t}^{2}(z, t)-b_{3}(z, t) u_{t}^{3}(z, t)+f_{0}(z, t),
\end{aligned}
$$

where

$$
\begin{aligned}
& a_{1}(z, t)=\left(c_{1}(z, t)+3 c_{2}(z, t) \vartheta_{d}^{2}(z, t)\right), \\
& a_{3}(z, t)=c_{2}(z, t) u^{3}(z, t), a_{2}(z, t)=3 c_{2}(z, t) \vartheta_{d}, \\
& b_{1}(z, t)=\left(d_{1}(z, t)+3 d_{2}(z, t) v_{d}^{2}(z, t)\right), \\
& b_{2}(z, t)=3 d_{2}(z, t) \vartheta_{d}, b_{3}(z, t)=d_{2}(z, t), \\
& f_{0}(z, t)=c_{1}(z, t) \vartheta_{d}(z, t)+c_{2}(z, t) \vartheta_{d}^{3}(z, t) .
\end{aligned}
$$

The stochastic part $q_{s}(\bullet)$ of $q(\bullet)$ is defined by

$$
\begin{aligned}
q_{s}(\bullet) & =c_{2}(z, t) u^{2}(z, t)\left[3 \vartheta_{s}(z, t)\right]-c_{2}(z, t) u(z, t)\left[3 \vartheta_{s}(z, t)\left(2 \vartheta_{d}(z, t)+\vartheta_{s}(z, t)\right)\right]+d_{2}(z, t) u_{t}^{2}(z, t)\left[3 v_{s}(z, t)\right] \\
& -d_{2}(z, t) u_{t}(z, t)\left[3 v_{s}(z, t)\left(2 v_{d}(z, t)+v_{s}(z, t)\right)\right]+c_{1}(z, t)\left[\vartheta_{s}(z, t)\right]+c_{2}(z, t)\left[3 \vartheta_{d}^{2}(z, t) \vartheta_{s}(z, t)+3 \vartheta_{d}(z, t) \vartheta_{s}^{2}(z, t)\right. \\
& \left.+\vartheta_{s}^{3}(z, t)\right]+d_{2}(z, t)\left[3 v_{d}^{2}(z, t) v_{s}(z, t)+3 v_{d}(z, t) v_{s}^{2}(z, t)+v_{s}^{3}(z, t)\right] .
\end{aligned}
$$

We now use formal time derivatives $\dot{W}_{i}(z, t), i=1, \ldots, 7$ of Wiener processes $W_{i}(z, t)$ with the spatial parameter $z$ and with different covariances to model the stochastic terms defined in the square brackets in (7). As such, we can rewrite (7) as

$$
\begin{aligned}
q_{s}(\bullet) & =\sum_{i=1}^{7} g_{i}\left(z, t, u(z, t), u_{t}(z, t)\right) \dot{W}_{i}(z, t) \\
& :=\boldsymbol{g}_{N}^{T}\left(z, t, u(z, t), u_{t}(z, t)\right) \dot{\boldsymbol{W}}(z, t),
\end{aligned}
$$

where $\boldsymbol{g}_{N}\left(z, t, u(z, t), u_{t}(z, t)\right)=\operatorname{col}\left(g_{i}\left(z, t, u(z, t), u_{t}(z, t)\right)\right)$ and $\boldsymbol{W}(z, t):=\operatorname{col}\left(W_{i}(z, t)\right), i=1, \ldots, q$ with $q=7$, and

$$
\begin{aligned}
& g_{1}=c_{2}(z, t) u^{2}(z, t), \quad g_{2}=-c_{2}(z, t) u(z, t), \\
& g_{3}=d_{2}(z, t) u_{t}^{2}(z, t), \quad g_{4}=-d_{2}(z, t) u_{t}(z, t), \\
& g_{5}=c_{1}(z, t), \quad g_{6}=c_{2}(z, t), \quad g_{7}=d_{2}(z, t),
\end{aligned}
$$


and for uniform notation we have defined $W_{5}(z, t)=W_{1}(z, t)$. The covariance matrix function of the $q$-dimensional $W(z, t)$ Wiener process is given by

$$
\mathbb{E}\left\{\langle\boldsymbol{h}(x, t), \boldsymbol{W}(x, t)\rangle_{L_{q}^{2}([0, L])}\langle\boldsymbol{h}(y, t), \boldsymbol{W}(y, t)\rangle_{L_{q}^{2}([0, L])}\right\}=\int_{0}^{L}\langle\boldsymbol{h}(x, t), \boldsymbol{Q}(x, y) \boldsymbol{h}(y, s)\rangle_{L_{q}^{2}([0, L])} d y\left(t-t_{0}\right) \wedge\left(s-t_{0}\right)
$$

for $t \geq t_{0}, s \geq t_{0}$, and any $q$-dimensional square integrable function $\boldsymbol{h}(x, t)$ on $[0, L]$, where $\boldsymbol{Q}(x, y)$ is a $q \times$ $q$ symmetric positive definite matrix function, and $\langle\cdot, \cdot\rangle_{L_{q}^{2}[[0, L])}$ is the inner product: $\langle\boldsymbol{h}(x, t), \boldsymbol{h}(x, s)\rangle_{L_{q}^{2}([0, L])}=$ $\int_{0}^{L} \boldsymbol{h}^{T}(x, t) \boldsymbol{h}(x, s) d x$.

Substituting (8) into (4), then into (1) results in the following stochastic riser system:

$$
\begin{aligned}
& \varrho a u_{t t}(z, t)=-E I u_{z z z z}(z, t)+\left(P_{0}+\frac{3 E a}{2} u_{z}^{2}(z, t)\right) u_{z z}(z, t)+f\left(z, t, u(z, t), u_{t}(z, t)\right)+\boldsymbol{g}_{N}\left(z, t, u(z, t), u_{t}(z, t)\right) \dot{\boldsymbol{W}}(z, t), \\
& -E I u_{z z z}(L, t)+P_{0} u_{z}(L, t)+\frac{E a}{2} u_{z}^{3}(L, t)=\varphi_{B}, \\
& u_{z z}(0, t)=0, u_{z z}(L, t)=0, u(0, t)=0, \\
& u\left(z, t_{0}\right)=u_{0}(z), u_{t}\left(z, t_{0}\right)=u_{1}(z) .
\end{aligned}
$$

Remark 2.1. If the term $\dot{W}(z, t)$ was assumed to be bounded, then the riser system (11) becomes a deterministic one and was considered in Stanway and Burrows (1981); Fard and Sagatun (2001); Fung et al. (1999); Fung and Tseng (1999); Do and Pan (2008a, 2009); Do (2011); Ge et al. (2010); He et al. (2011); Nguyen et al. (2013); Bohm et al. (2014); Torres et al. (2015), where the term $\dot{W}(z, t)$ was taken as a summation of several sinusoidal functions of random signals.

\subsection{Control objectives}

Before stating the control objective, we make the following assumption.

\section{Assumption 2.1.}

1. The initial values $\left(u_{0}(z), u_{1}(z)\right)$ are bounded in $L^{2}([0, L])$, i.e., there exist nonnegative constants $\epsilon_{10}$ and $\epsilon_{11}$ such that

$$
\int_{0}^{L} u_{0}^{2}(z) d z \leq \epsilon_{10}, \quad \int_{0}^{L} u_{1}^{2}(z) d z \leq \epsilon_{11} .
$$

2. The restoring coefficients $\left(c_{1}(z, t), c_{2}(z, t)\right)$, and damping coefficients $\left(d_{1}(z, t), d_{2}(z, t)\right)$ are positive and bounded in $L^{2}([0, L])$. The Wiener processes $W_{i}(z, t), i=1, \ldots, 7$ with the covariance $Q_{i}(z, t)$ are independent. The covariances $Q_{i}(z, t)$, the membrane displacement $\vartheta_{d}(z, t)$ and fluid/air velocity $v_{d}(z, t)$ are also bounded in $L^{2}([0, L])$.

Remark 2.2. In particular, Assumption 2.1.1 only requires the initial values be bounded in $L^{2}$-norm instead of a point-wise norm while 2.1.2 always holds in practice.

This paper addresses the following control objective.

Control Objective 2.1. Under Assumption 2.1, design the boundary control $\varphi_{B}$ as a function of $u(L, t)$ and $u_{t}(L, t)$ such that the stochastic riser system (11) is almost sure globally practically stable at the origin.

\section{Well-posedness and Stability of Nonlinear Stochastic Evolution Systems}

This section presents results on well-posedness and stability of nonlinear stochastic evolution systems. These results will be used in the control design and stability analysis of the stochastic riser system in sequel. The material in this section is somewhat dry but has rewarding applications in the control design and stability and stability analysis in the next section. 


\subsection{Space notations}

Let $H$ be a separable Hilbert space with inner product $\langle., .\rangle_{H}$ and norm $\|.\|_{H}$, and $H^{*}$ be its dual space by the Riesz isomorphism. Let $V$ be a real reflexible Banach space such that $V \subset H$ continuously and densely. Then for its dual space $V^{*}$, it follows that $H^{*} \subset V^{*}$. From the definitions of $H$ and $V$, we have that

$$
V \subset H \equiv H^{*} \subset V^{*}
$$

continuously and densely. Let the dualization between $V$ and $V^{*}$, in which the norm is denoted by $\|\cdot\|_{V^{*}}$, be denoted by $\langle., .\rangle_{V, V^{*}}$ (i.e., $\langle z, v\rangle_{V, V^{*}}=z(v)$ for $\left.z \in V^{*}, v \in V\right)$. The dualization between $V$ and $V^{*}$, in which the norm is denoted by $\|.\|_{V^{*}}$, is denoted by $\langle., .\rangle_{V, V^{*}}$ (i.e., $\langle z, v\rangle_{V, V^{*}}=z(v)$ for $\left.z \in V^{*}, v \in V\right)$, we then have

$$
\langle z, v\rangle_{V, V^{*}}=\langle z, v\rangle_{H}, u \in H, v \in V .
$$

For $\boldsymbol{u}=\operatorname{col}\left(\boldsymbol{u}_{1}, \boldsymbol{u}_{2}\right)$ with $\boldsymbol{u}_{1} \in V$ and $\boldsymbol{u}_{2} \in H$, we denote the space $\mathcal{H}=V \times H$ for $\boldsymbol{u}$ equipped with the norm $\|\boldsymbol{u}\|_{\mathcal{H}}^{2}:=\left\|\boldsymbol{u}_{1}\right\|_{V}^{2}+\left\|\boldsymbol{u}_{2}\right\|_{H}^{2}$. Also, let $\mathcal{V}$ be a real reflexible Banach space such that $\mathcal{V} \subset \mathcal{H}$ continuously and densely. Then for its dual space $\mathcal{V}^{*}$, it follows that $\mathcal{V} \subset \mathcal{H} \subset \mathcal{V}^{*}$.

Let $\boldsymbol{W}(t), t \geq t_{0}$ be a cylindrical $\boldsymbol{Q}$-Wiener process defined on a separable Hilbert space $K$ with respect to a probability space $(\Omega, \mathcal{F}, \mathbb{P})$ equipped with a filtration $\mathcal{F}_{t \geq t_{0}}$ satisfying usual conditions (i.e., it is right continuous and $\mathcal{F}_{t_{0}}$ contains all $\mathbb{P}$-null sets). Assume that $e_{k}, k \in \mathbb{K}$ is an orthonormal basis of $K$ consisting of eigenvectors of $\boldsymbol{Q}$ with corresponding eigenvalues $\lambda_{k}>0, k \in \mathbb{K}$, numbered in a decreasing order. Then we have, see Prato and Zabczyk (1992):

$$
\boldsymbol{W}(t)=\sum_{k \in \mathbb{K}} \sqrt{\lambda_{k}} \beta_{k}(t) e_{k},
$$

where $\beta_{k}(t)$ is a sequence of real-valued standard Brownian motions that are mutually independent on the probability space $(\Omega, \mathcal{F}, \mathbb{P})$.

Let $\boldsymbol{K}_{0}=\boldsymbol{Q}^{\frac{1}{2}}$ and $\mathscr{L}_{2}^{0}=\left(\boldsymbol{K}_{0}, H\right)$, which is the space of all Hilbert-Schmidt operators from $\boldsymbol{K}_{0}$ into $H$. The space $\mathscr{L}_{2}^{0}$ is a separable Hilbert space equipped with the norm $\|\boldsymbol{\Phi}\|_{\mathscr{L}_{2}^{0}}^{2}=\operatorname{Tr}\left(\boldsymbol{\Phi} Q \boldsymbol{\Phi}^{\star}\right)$ for $\boldsymbol{\Phi} \in \mathscr{L}_{2}^{0}$, where $\operatorname{Tr}(\cdot)$ stands for the trace operator of (.) and $\star$ denotes the adjoint operation. We denote by $\mathscr{L}(K, H)$ the set of all linear bounded operators from $K$ into $H$. We also denote by $\mathcal{J}^{p}\left(\left[t_{0}, T\right] ; V\right)$ the space of all $V$-valued process $\boldsymbol{u}(t)$, which are $\mathcal{F}_{t}$-measurable from $\left[t_{0}, T\right]$ to $V$ and satisfy $\mathbb{E}\left\{\int_{t_{0}}^{T}\|\boldsymbol{u}(t)\|^{p} d t<\infty\right\}$.

\subsection{Stochastic evolution systems}

Let us consider the following nonlinear SES on the space $\mathcal{H}$ :

$$
\begin{aligned}
& d \boldsymbol{X}(t)=\boldsymbol{F}(\boldsymbol{X}(t), t) d t+\boldsymbol{G}(\boldsymbol{X}(t), t) d \boldsymbol{W}(t), \\
& \boldsymbol{X}\left(t_{0}\right)=\boldsymbol{X}_{0} \in \mathcal{H},
\end{aligned}
$$

where $\boldsymbol{X}=\operatorname{col}\left(\boldsymbol{X}_{1}, \boldsymbol{X}_{2}\right)$ with $\boldsymbol{X}_{1}$ and $\boldsymbol{X}_{2}$ being the $n$-dimensional state vector; $\boldsymbol{X}_{0}=\operatorname{col}\left(\boldsymbol{X}_{10}, \boldsymbol{X}_{20}\right)$; and

$$
\begin{aligned}
& \boldsymbol{A}(t)=\left[\begin{array}{cc}
0 & \boldsymbol{I} \\
\boldsymbol{A}_{1 L}(t) & \boldsymbol{A}_{2 L}(t)
\end{array}\right], \boldsymbol{G}(\boldsymbol{X}, t)=\left[\begin{array}{c}
0 \\
\boldsymbol{G}_{N}(\boldsymbol{X}, t)
\end{array}\right], \\
& \boldsymbol{F}(\boldsymbol{X}, t)=\boldsymbol{A}(t) \boldsymbol{X}+\left[\begin{array}{c}
0 \\
\boldsymbol{F}_{N}(\boldsymbol{X}, t)
\end{array}\right] .
\end{aligned}
$$

In (17), $\boldsymbol{A}_{i L}(t), i=1,2$ are a continuous family of closed random linear operators; $\boldsymbol{F}_{N}: \mathcal{H} \times\left[t_{0}, \infty\right) \rightarrow V^{*}$ and $\boldsymbol{G}_{N}: \mathcal{H} \times\left[t_{0}, \infty\right) \rightarrow \mathscr{L}_{2}\left(K_{0}, H\right)$ are Borel measurable, and $\boldsymbol{W}$ is a $Q$-Wiener process. We first define of the variational solution of (16) in the following definition.

Definition 3.1. An $\mathcal{F}_{t}$-measurable $\mathcal{H}$-valued stochastic process $\left\{X(t), t \in\left[t_{0}, T\right]\right\}$ is said to be a variational solution of (16) if for any $\boldsymbol{\phi}, \boldsymbol{\psi} \in V$ the following conditions hold:

$$
\begin{aligned}
\left\langle\boldsymbol{X}_{1}(t), \boldsymbol{\phi}\right\rangle_{H} & =\left\langle\boldsymbol{X}_{10}, \boldsymbol{\phi}\right\rangle_{H}+\int_{t_{0}}^{t}\left\langle\boldsymbol{X}_{1}(s), \boldsymbol{\phi}\right\rangle_{H} d s, \\
\left\langle\boldsymbol{X}_{2}(t), \boldsymbol{\psi}\right\rangle_{H} & =\left\langle\boldsymbol{X}_{20}, \boldsymbol{\psi}\right\rangle_{H}+\int_{t_{0}}^{t}\left\langle\boldsymbol{G}_{N}(\boldsymbol{X}(s), s) d \boldsymbol{W}(s), \boldsymbol{\psi}\right\rangle_{H}+\int_{t_{0}}^{t}\left\langle\boldsymbol{A}_{1 L}(s) \boldsymbol{X}_{1}(s)+\boldsymbol{A}_{2 L}(s) \boldsymbol{X}_{2}(s)+\boldsymbol{F}_{N}(\boldsymbol{X}(s), s), \boldsymbol{\psi}\right\rangle_{V, V^{*}} d s
\end{aligned}
$$

for each $t \in\left[t_{0}, T\right]$. If $T$ is replaced by $\infty$, then $\boldsymbol{X}(t), t \geq t_{0}$, is said to be a global variational solution of (16). 
We now define a.s. sure stability, a.s. asymptotic stability, and a.s. practical stability of the strong solution of the system (16).

Definition 3.2. The strong solution of (16) is said to be:

1) a.s. globally stable if for any constant $\varepsilon>0$, there exists a class $\mathcal{K}$ function $\gamma(\cdot)$ such that $\mathbb{P}\left\{\|X(t)\|_{\mathcal{H}}<\right.$ $\left.\gamma\left(\left\|\boldsymbol{X}_{0}\right\|_{\mathcal{H}}\right)\right\} \geq 1-\varepsilon$ for all $\boldsymbol{X}_{0} \in \mathcal{H} \backslash\{0\}$

2) a.s. globally asymptotically stable if it is almost sure globally stable and $\mathbb{P}\left\{\lim _{t \rightarrow \infty}\|X(t)\|_{\mathcal{H}}=0\right\}=1$ for all $\boldsymbol{X}_{0} \in \mathcal{H}$; and

3) a.s. sure globally practically stable if $\mathbb{P}\left\{\lim _{t \rightarrow \infty}\|X(t)\|_{\mathcal{H}} \leq \varepsilon\right\}=1$ for all $\boldsymbol{X}_{0} \in \mathcal{H}$, where $\varepsilon$ is a nonnegative constant.

\subsection{The Itô formula} lemma.

We here present the Itô formula, which will play an important role in the rest of the paper in the following

Lemma 3.1. (The Itô formula) Let $C^{2}\left(\left[t_{0}, \infty\right) ; \mathcal{H}\right)$ denote the space of all real-valued nonnegative functions $U(X, t)$ on $\mathcal{H}$ with the following properties:

1. $U(\boldsymbol{X}, t)$ is twice (Fréchet) differentiable in $\boldsymbol{X}$ and is differentiable in $t$.

2. Both $U_{\boldsymbol{X}}(\boldsymbol{X}, t)$ and $U_{t}(\boldsymbol{X}, t)$ are continuous in $\mathcal{H}$ and $U_{\boldsymbol{X} X}(\boldsymbol{X}, t)$ is continuous in $\mathscr{L}(\mathcal{H}, \mathcal{H})$.

Suppose that $\boldsymbol{X}(t), t \in\left[t_{0}, \infty\right)$, is a solution of (16). Then

$$
U(\boldsymbol{X}, t)=U\left(\boldsymbol{X}_{0}, t_{0}\right)+\int_{t_{0}}^{t} \mathcal{L} U(\boldsymbol{X}(s), s) d s+\int_{t_{0}}^{t}\left\langle U_{\boldsymbol{X}}(\boldsymbol{X}(s), s), \boldsymbol{G}(\boldsymbol{X}(s), s) d \boldsymbol{W}(s)\right\rangle_{\mathcal{H}},
$$

where the infinite generator $\mathcal{L} U(\boldsymbol{X}, t)$ is given by

$$
\mathcal{L} U(\boldsymbol{X}, t)=U_{t}(\boldsymbol{X}, t)+\left\langle\boldsymbol{F}(\boldsymbol{X}, t), U_{\boldsymbol{X}}(\boldsymbol{X}, t)\right\rangle_{\mathcal{H}}+\frac{1}{2} \operatorname{Tr}\left(U_{\boldsymbol{X} \boldsymbol{X}}(\boldsymbol{X}, t) \boldsymbol{G}(\boldsymbol{X}, t) \boldsymbol{Q} \boldsymbol{G}^{\star}(\boldsymbol{X}, t)\right) .
$$

\subsection{Existence and uniqueness theorem for nonlinear stochastic evolution systems}

This subsection gives a global existence and uniqueness result of the variational solution of (16). We impose the following conditions.

\section{Assumption 3.1.}

1) $\boldsymbol{A}_{i L}(t), i=1,2$ are a continuous family of closed random linear operators with the domain $\mathcal{D}\left(\boldsymbol{A}_{i L}\right)$ dense in $H$ such that $\boldsymbol{A}_{i L}: V \rightarrow V^{*}$, and for any $\boldsymbol{X}_{1} \in V$ and $\boldsymbol{X}_{2} \in H, \boldsymbol{A}_{1 L}(t) \boldsymbol{X}_{1}$ and $\boldsymbol{A}_{2 L}(t) \boldsymbol{X}_{2}$ are adapted continuous $V^{*}-$ and $H$-valued processes, respectively.

2) Let us write $\boldsymbol{F}_{N}(\boldsymbol{X}, t)$ and $\boldsymbol{G}_{N}(\boldsymbol{X}, t)$ as

$$
\begin{aligned}
& \boldsymbol{F}_{N}(\boldsymbol{X}, t)=\boldsymbol{F}_{0}(t)+\hat{\boldsymbol{F}}_{N}(\boldsymbol{X}, t), \\
& \boldsymbol{G}_{N}(\boldsymbol{X}, t)=\boldsymbol{G}_{0}(t)+\hat{\boldsymbol{G}}_{N}(\boldsymbol{X}, t),
\end{aligned}
$$

and consider the linearized system of (16), i.e.,

$$
\begin{aligned}
& d \boldsymbol{X}_{1}(t)=\boldsymbol{X}_{2}(t), \\
& d \boldsymbol{X}_{2}(t)=\left[\boldsymbol{A}_{1 L}(t) \boldsymbol{X}_{1}(t)+\boldsymbol{A}_{2 L}(t) \boldsymbol{X}_{2}(t)+\boldsymbol{F}_{0}(t)\right] d t+\boldsymbol{G}_{0}(t) d \boldsymbol{W}(t), \\
& \boldsymbol{X}_{1}\left(t_{0}\right):=\boldsymbol{X}_{10} \in V, \quad \boldsymbol{X}_{2}\left(t_{0}\right):=\boldsymbol{X}_{20} \in H .
\end{aligned}
$$

For the system (22), define the function $U_{L}\left(\boldsymbol{X}_{1}, \boldsymbol{X}_{2}\right)$ as

$$
U_{L}\left(\boldsymbol{X}_{1}, \boldsymbol{X}_{2}\right)=\left\|\boldsymbol{X}_{1}\right\|_{V}^{2}+\left\|\boldsymbol{X}_{2}\right\|_{H}^{2} .
$$

There exists a constant $b_{1}$ such that

$$
\mathbb{E}\left\{\sup _{t_{0} \leq \tau \leq t} U_{L}\left(\boldsymbol{X}_{1}(\tau), \boldsymbol{X}_{2}(\tau)\right)\right\} \leq b_{1} \mathbb{E}\left\{\left\|\boldsymbol{X}_{10}\right\|_{V}^{2}+\left\|\boldsymbol{X}_{20}\right\|_{H}^{2}+\int_{t_{0}}^{t}\left(\left\|\boldsymbol{F}_{0}(s)\right\|_{H}^{2}+\operatorname{Tr}\left(\boldsymbol{G}_{0}(s) \boldsymbol{Q}(s) \boldsymbol{G}_{0}^{\star}(s)\right)\right) d s\right\}
$$

a.e. $(t, \omega) \in\left[t_{0}, \infty\right) \times \Omega$. 
Next, we assume that $\boldsymbol{F}_{N}: \mathcal{H} \times\left[t_{0}, \infty\right) \rightarrow V^{*}$ and $\boldsymbol{G}_{N}: \mathcal{H} \times\left[t_{0}, \infty\right) \rightarrow \mathscr{L}_{2}\left(\boldsymbol{K}_{0}, \mathcal{H}\right)$ are Borel measurable and satisfy the following local growth and local Lipschitz conditions.

\section{Assumption 3.2.}

1) For any $\boldsymbol{u} \in V, \boldsymbol{v} \in H$ with $\|\boldsymbol{u}\|_{V} \vee\|\boldsymbol{v}\|_{H} \leq \varepsilon$, where $\varepsilon$ is a positive constant, there exist constants $b_{2}$ and $b_{3}$ such that

$$
\begin{aligned}
& \mathbb{E}\left\{\int_{t_{0}}^{T}\left(\left\|\boldsymbol{F}_{N}(0,0, t)\right\|_{H}^{2}+\left\|\boldsymbol{G}_{N}(0,0, t)\right\|_{\mathscr{L}_{2}^{0}}^{2}\right) d t\right\} \leq b_{2}, \\
& \left\|\tilde{\boldsymbol{F}}_{N}(\boldsymbol{u}, \boldsymbol{v}, t)\right\|_{H}^{2}+\left\|\tilde{\boldsymbol{G}}_{N}(\boldsymbol{u}, \boldsymbol{v}, t)\right\|_{\mathscr{L}_{2}^{0}}^{2} \leq b_{3}\left(1+\|\boldsymbol{u}\|_{V}^{2}+\|\boldsymbol{v}\|_{H}^{2}\right), \text { a.e. }(t, \omega) \in\left[t_{0}, \infty\right) \times \Omega,
\end{aligned}
$$

where $\tilde{\boldsymbol{F}}_{N}(\boldsymbol{u}, \boldsymbol{v}, t)=\boldsymbol{F}_{N}(\boldsymbol{u}, \boldsymbol{v}, t)-\boldsymbol{F}_{N}(0,0, t)$ and $\tilde{\boldsymbol{G}}_{N}(\boldsymbol{u}, \boldsymbol{v}, t)=\boldsymbol{G}_{N}(\boldsymbol{u}, \boldsymbol{v}, t)-\boldsymbol{G}_{N}(0,0, t)$.

2) For any $\boldsymbol{u}_{1}, \boldsymbol{u}_{2} \in V$ and $\boldsymbol{v}_{1}, \boldsymbol{v}_{2} \in H$ with $\left\|\boldsymbol{u}_{1}\right\|_{V} \vee\left\|\boldsymbol{u}_{2}\right\|_{V} \vee\left\|\boldsymbol{v}_{1}\right\|_{H} \vee\left\|\boldsymbol{v}_{2}\right\|_{H} \leq \varepsilon$, there exists a constant $b_{4}$ such that

$$
\begin{aligned}
& \left\|\boldsymbol{F}_{N}\left(\boldsymbol{u}_{1}, \boldsymbol{v}_{1}, t\right)-\boldsymbol{F}_{N}\left(\boldsymbol{u}_{2}, \boldsymbol{v}_{2}, t\right)\right\|_{H}^{2}+\left\|\boldsymbol{G}_{N}\left(\boldsymbol{u}_{1}, \boldsymbol{v}_{1}, t\right)-\boldsymbol{G}_{N}\left(\boldsymbol{u}_{2}, \boldsymbol{v}_{2}, t\right)\right\|_{\mathscr{L}_{2}^{0}}^{2} \leq b_{4}\left(\left\|\boldsymbol{u}_{1}-\boldsymbol{u}_{2}\right\|_{V}^{2}+\left\|\boldsymbol{v}_{1}-\boldsymbol{v}_{2}\right\|_{H}^{2}\right), \\
& \quad \text { a.e. }(t, \omega) \in\left[t_{0}, \infty\right) \times \Omega .
\end{aligned}
$$

Remark 3.1. It is observed that only local conditions listed in Assumptions 3.1 and 3.2 are required. These Assumptions together with the Lyapunov conditions stated in Theorem 3.1 below will ensure global existence and uniqueness of the variational solution of the SES (16). These Assumptions and conditions are much more relaxed than those global conditions required in literature, see Assumptions in Appendix A.

Under the above local conditions stated in Assumptions 3.1 and 3.2, the following theorem gives the global existence and uniqueness of the variational solution of (16).

Theorem 3.1. Under Assumptions 3.1 and 3.2, suppose further that there exists a function $U(\boldsymbol{X}, t) \in C^{2}\left(\mathcal{H} ;\left[t_{0}, \infty\right)\right)$ referred to as a Lyapunov function and a positive constant $c$ such that

1. Growth condition on $\mathcal{L} U(\boldsymbol{X}, t)$ :

$$
\mathcal{L} U(\boldsymbol{X}, t) \leq c(1+U(\boldsymbol{X}, t)), \quad \boldsymbol{X} \in V, t \in\left[t_{0}, \infty\right),
$$

2. Radially unbounded condition on $U(\boldsymbol{X}, t)$ :

$$
\lim _{\|\boldsymbol{X}\|_{\mathcal{H}} \rightarrow \infty} U(\boldsymbol{X}, t)=\infty, \quad \boldsymbol{X} \in V, t \in\left[t_{0}, \infty\right) .
$$

Then, for any initial data $\boldsymbol{X}_{0} \in \mathcal{H}$ with $\mathbb{E}\left\{\left\|\boldsymbol{X}_{0}\right\|_{\mathcal{H}}^{2}\right\}<\infty$, the system (16) has a unique global variational solution.

Proof. See Appendix A.

\subsection{An a.s. stability theorem for nonlinear SESS}

We here provide a theorem that includes a.s. LaSalle-type, a.s. Lyapunov-type, and a.s. practical stability theorems.

Theorem 3.2. Under Assumptions 3.1 and 3.2, assume further that there exists a function $U(\boldsymbol{X}, t) \in C^{2}\left(\mathcal{H} ;\left[t_{0}, \infty\right)\right)$ referred to as a Lyapunov function; and class $\mathcal{K}_{\infty}$ functions $\alpha_{1}$ and $\alpha_{2}$ such that for all $\boldsymbol{X} \in \mathcal{V}$ and $t \in\left[t_{0}, \infty\right)$

$$
\alpha_{1}\left(\|\boldsymbol{X}\|_{\mathcal{H}}\right) \leq U(\boldsymbol{X}, t) \leq \alpha_{2}\left(\|\boldsymbol{X}\|_{\mathcal{H}}\right)
$$

1) [LaSalle-type theorem] Suppose that there exists a continuous and nonnegative function $M: \mathcal{H} \rightarrow \mathbb{R}$ such that

$$
\mathcal{L} U \leq-M(X)
$$

Then the system (16) has a unique variational solution for each $\boldsymbol{X}_{0} \in \mathcal{H}$, the equilibrium $\boldsymbol{X} \equiv 0$ is a.s. globally stable, and

$$
\mathbb{P}\left\{\lim _{t \rightarrow \infty} M(X(t))=0\right\}=1, \forall X_{0} \in \mathcal{H} .
$$


2) [Lyapunov-type theorem] Suppose that there exists a class $\mathcal{K}$ function $\alpha_{3}$ such that for all $\boldsymbol{X} \in \mathcal{V}$ and $t \in\left[t_{0}, \infty\right)$

$$
\mathcal{L} U \leq-\alpha_{3}\left(\|\boldsymbol{X}\|_{\mathcal{H}}\right)
$$

Then the equilibrium $\boldsymbol{X} \equiv 0$ is a.s. globally asymptotically stable.

3) [Practical stability theorem] Suppose that there exists a class $\mathcal{K}_{\infty}$ function $\alpha_{3}$ such that for all $\boldsymbol{X} \in \mathcal{V}$ and $t \in\left[t_{0}, \infty\right)$

$$
\mathcal{L} U \leq-\alpha_{3}\left(\|X\|_{\mathcal{H}}\right)+\varepsilon_{0},
$$

where $\varepsilon_{0}$ is a nonnegative constant. Then the equilibrium $\boldsymbol{X} \equiv 0$ is a.s. practically stable.

Proof. See Appendix B.

\section{Control design}

\subsection{Abstract formulation}

Introduce the functional spaces: $H=L^{2}([0, L]), V=H^{2}([0, L])$, and $V^{*}=H^{-2}([0, L])$ (the dual of the space $V)$, where $H^{2}([0, L])$ and $H^{-2}([0, L])$ are Sobolev spaces. Define $\mathcal{H}=V \times H, \mathcal{V}=H_{0}^{2}([0, L]) \times L^{2}([0, L])$, where $H_{0}^{2}([0, L])$ is a Sobolev space $H^{2}([0, L])$ satisfying certain boundary conditions to be specified, and $\mathcal{V}^{*}=$ $H^{-2}([0, L]) \times L^{2}([0, L])$ (the dual of the space $\left.\mathcal{V}\right)$. Then, we have the embedding $\mathcal{V} \subset \mathcal{H} \subset \mathcal{V}^{*}$. We denote by $\langle., .\rangle_{\mathcal{H}}$ and $\langle., .\rangle_{\mathcal{V}, \mathcal{V}^{*}}$ the inner product in $\mathcal{H}$ and the dualization between $\mathcal{V}$ and $\mathcal{V}^{*}$, respectively; by $\|.\|_{\mathcal{H}}$ and $\|.\|_{\mathcal{V}}$ the norms in $\mathcal{H}$ and $\mathcal{V}$, respectively. Then, we have $\langle z, v\rangle_{\mathcal{V}, \mathcal{V}^{*}}=\langle z, v\rangle_{\mathcal{H}}$ for $\boldsymbol{u} \in \mathcal{H}, \boldsymbol{v} \in \mathcal{V}$, and $\|\operatorname{col}(\boldsymbol{u}, \boldsymbol{v})\|_{\mathcal{H}}^{2}=\|\boldsymbol{u}\|_{V}^{2}+\|\boldsymbol{v}\|_{H}^{2}$ for $\boldsymbol{u} \in V$ and $\boldsymbol{v} \in H$.

Considering $z \in[0, L]$ as the parameter defined at every $t \geq t_{0}$, we can regard $u(z, t), u_{t}(z, t)$, and $\boldsymbol{W}(z, t)=$ $\operatorname{col}\left(W_{i}(z, t)\right), i=1, \ldots, 7$ as $X_{1}(t)$ in $H^{2}([0, L]), X_{2}(t)$ in $L^{2}([0, L])$, and $\boldsymbol{W}(t) \in L^{2}([0, L])$, respectively. Similarly, $u(0, t)$ and $u(L, t)$ are regarded as $X_{1}^{B 0}(t) \in \mathbb{R}$ and $X_{1}^{B L}(t) \in \mathbb{R}$, respectively, and so on. Let us define operators $A_{i L}(t), i=1,2$ and $A_{B L}(t)$ as follows:

$$
\begin{aligned}
& A_{1 L}(t) X_{1}=\frac{1}{\varrho a}\left(-E I \mathbb{D}^{4} X_{1}+P_{0} \mathbb{D}^{2} X_{1}-a_{1}(t) X_{1}-b_{1}(t) X_{2}\right), \\
& A_{2 L}(t) X_{2}=-\frac{1}{\varrho a} b_{1}(t) X_{2}, \\
& A_{B L}(t) X_{1}^{L}=-E I \mathbb{D}^{3} X_{1}^{B L}+P_{0} \mathbb{D} X_{1}^{B L},
\end{aligned}
$$

where $(\mathbb{D} \phi)(z):=\frac{\partial \phi(z)}{\partial z}$. We now can write the stochastic riser system (11) in the following abstract form:

$$
\begin{aligned}
& d X_{1}=X_{2} d t, \\
& d X_{2}=\left[A_{1 L}(t) X_{1}+A_{2 L}(t) X_{2}+F_{N}\left(X_{1}, X_{2}, t\right)\right] d t+g_{N}^{T}\left(X_{1}, X_{2}, t\right) d \boldsymbol{W}(t), \\
& X_{1}\left(t_{0}\right):=X_{10}=u_{0}, X_{2}\left(t_{0}\right):=X_{20}=u_{1},
\end{aligned}
$$

subject to the boundary conditions

$$
\begin{aligned}
& X_{1}^{B 0}=0, \mathbb{D}^{2} X_{1}^{B 0}=0, \mathbb{D}^{2} X_{1}^{B L}=0 \\
& A_{B L}(t) X_{1}^{B L}+F_{B N}\left(X_{1}^{B L}, X_{2}^{B L}, t\right)=0
\end{aligned}
$$

where

$$
\begin{aligned}
& F_{N}\left(X_{1}, X_{2}, t\right)=\frac{1}{\varrho a}\left[a_{2}(t) X_{1}^{2}-a_{3}(t) X_{1}^{3}+b_{2}(t) X_{2}^{2}-b_{3}(t) X_{2}^{3}+\frac{3 E a}{2}\left(\mathbb{D} X_{1}\right)^{2} \mathbb{D}^{2} X_{1}+f_{0}(t)\right], \\
& F_{B N}\left(X_{1}^{B L}, X_{2}^{B L}, t\right)=\frac{E a}{2}\left(\mathbb{D} X_{1}^{B L}\right)^{3}-\varphi_{B} .
\end{aligned}
$$




\subsection{Design of boundary control $\varphi_{B}$}

To design the boundary control $\varphi_{B}$, we consider the following Lyapunov function candidate:

$$
U=\frac{\varrho a}{2}\left\|X_{2}\right\|_{H}^{2}+\frac{P_{0}}{2}\left\|\mathbb{D} X_{1}\right\|_{H}^{2}+\frac{E a}{8}\left\|\left(\mathbb{D} X_{1}\right)^{2}\right\|_{H}^{2}+\frac{E I}{2}\left\|\mathbb{D}^{2} X_{1}\right\|_{H}^{2}+\gamma \varrho a\left\langle X_{1}, X_{2}\right\rangle_{H},
$$

where the constant $\gamma$ satisfies

$$
0<\gamma<1 \wedge \frac{P_{0}}{4 L^{2} \varrho a} .
$$

Remark 4.1. The first term in the Lyapunov function candidate $U$ defined in (38) is the kinetic energy of the riser. The second, third and fourth terms are the potential energy (due to tension, axial deformation, bending). The last term (referred to as the cross term) is included to utilize the structural damping of the riser, see the negative definite terms with $\gamma$ as a factor in (43).

Let $\boldsymbol{X}=\operatorname{col}\left(X_{1}, X_{2}\right)$. Since $\mathcal{H}=V \times H$, and

$$
\begin{aligned}
\|\boldsymbol{X}\|_{\mathcal{H}}^{2} & =\left\|X_{1}\right\|_{V}^{2}+\left\|X_{2}\right\|_{H}^{2} \\
& =\left\|\mathbb{D}^{2} X_{1}\right\|_{H}^{2}+\left\|\mathbb{D} X_{1}\right\|_{H}^{2}+\left\|X_{1}\right\|_{H}^{2}+\left\|X_{2}\right\|_{H}^{2},
\end{aligned}
$$

an application of Poincare's inequality and the Sobolev embedding and interpolation theorems, see Adams and Fournier (2003), shows that

$$
\begin{aligned}
& U \geq \frac{\varrho a}{2}(1-\gamma)\left\|X_{2}^{2}\right\|_{H}^{2}+\left(\frac{P_{0}}{2}-2 \gamma \varrho a L^{2}\right)\left\|\mathbb{D} X_{1}\right\|_{H}^{2}+\frac{E a}{8}\left\|\left(\mathbb{D} X_{1}\right)^{2}\right\|_{H}^{2}+\frac{E I}{2}\left\|\mathbb{D}^{2} X_{1}\right\|_{H}^{2} \geq \alpha_{1}\left(\|X\|_{\mathcal{H}}\right), \\
& U \leq \frac{\varrho a}{2}(1+\gamma)\left\|X_{2}^{2}\right\|_{H}^{2}+\left(\frac{P_{0}}{2}+2 \gamma \varrho a L^{2}\right)\left\|\mathbb{D} X_{1}\right\|_{H}^{2}+\frac{E a}{8}\left\|\left(\mathbb{D} X_{1}\right)^{2}\right\|_{H}^{2}+\frac{E I}{2}\left\|\mathbb{D}^{2} X_{1}\right\|_{H}^{2} \leq \alpha_{2}\left(\|X\|_{\mathcal{H}}\right),
\end{aligned}
$$

where $\alpha_{1}$ and $\alpha_{2}$ are class $\mathcal{K}_{\infty}$ functions of $\|\boldsymbol{X}\|_{\mathcal{H}}$. The infinite generator $\mathcal{L} U$ along the solutions of (35) and (36) is

$$
\begin{aligned}
\mathcal{L} U & =\left\langle X_{2}, A_{1 L}(t) X_{1}+A_{2 L}(t) X_{2}+F_{N}\left(X_{1}, X_{2}, t\right)\right\rangle_{H}+E I\left\langle\mathbb{D}^{2} X_{1}, \mathbb{D}^{2} X_{2}\right\rangle_{H}+P_{0}\left\langle\mathbb{D} X_{1}, \mathbb{D} X_{2}\right\rangle_{H} \\
& +\frac{E a}{2}\left\langle\left(\mathbb{D} X_{1}\right)^{3}, \mathbb{D} X_{2}\right\rangle_{H}+\gamma \varrho a\left\|X_{2}\right\|_{H}^{2}+\gamma\left\langle X_{1}, A_{1 L}(t) X_{1}+A_{2 L}(t) X_{2}+F_{N}\left(X_{1}, X_{2}, t\right)\right\rangle_{H}+\frac{1}{2}\left\|\boldsymbol{Q}^{\frac{1}{2}}(t) \boldsymbol{g}_{N}\right\|_{H}^{2}
\end{aligned}
$$

Substituting $A_{1 L}(t) X_{1}$ and $A_{2 L}(t) X_{2}$ defined in (34) and $F_{N}\left(X_{1}, X_{2}, t\right)$ defined in (37) into (42), and using integration by parts result in

$$
\begin{aligned}
\mathcal{L} U & =-\gamma E I\left\|\mathbb{D}^{2} X_{1}\right\|_{H}^{2}-\gamma P_{0}\left\|\mathbb{D} X_{1}\right\|_{H}^{2}-\frac{\gamma E a}{2}\left\|\left(\mathbb{D} X_{1}\right)^{2}\right\|_{H}^{2}+\gamma \varrho a\left\|X_{2}\right\|_{H}^{2}-\gamma\left\|a_{1}^{\frac{1}{2}}(t) X_{1}\right\|_{H}^{2}-\gamma\left\|a_{3}^{\frac{1}{2}}(t) X_{1}^{2}\right\|_{H}^{2}-\left\|b_{1}^{\frac{1}{2}}(t) X_{2}\right\|_{H}^{2} \\
& -\left\|b_{3}^{\frac{1}{2}}(t) X_{2}^{2}\right\|_{H}^{2}+\varphi_{B}\left(\gamma X_{1}^{B L}+X_{2}^{B L}\right)+T,
\end{aligned}
$$

where

$$
\begin{aligned}
T & =-\left\langle\left(a_{1}(t)+\gamma b_{1}(t)\right) X_{1}, X_{2}\right\rangle_{H}+\left\langle a_{2}(t) X_{1}^{2}, X_{2}\right\rangle_{H}-\left\langle a_{3}(t) X_{1}^{3}, X_{2}\right\rangle_{H}+\left\langle b_{2}(t) X_{2}^{2}, X_{2}\right\rangle_{H}+\gamma\left\langle a_{2}(t) X_{1}^{2}, X_{1}\right\rangle_{H} \\
& +\gamma\left\langle b_{2}(t) X_{1}, X_{2}^{2}\right\rangle_{H}-\gamma\left\langle b_{3}(t) X_{1}, X_{2}^{3}\right\rangle_{H}+\left\langle\gamma X_{1}+X_{2}, f_{0}(t)\right\rangle_{H}+\left\|c_{2}(t) Q_{1}^{\frac{1}{2}}(t) X_{1}^{2}\right\|_{H}^{2}+\left\|c_{2}(t) Q_{2}^{\frac{1}{2}}(t) X_{1}\right\|_{H}^{2} \\
& +\left\|d_{2}(t) Q_{3}^{\frac{1}{2}}(t) X_{2}^{2}\right\|_{H}^{2}+\left\|d_{2}(t) Q_{4}^{\frac{1}{2}}(t) X_{2}\right\|_{H}^{2}+\left\|c_{1}(t) Q_{5}^{\frac{1}{2}}(t)\right\|_{H}^{2}+\left\|c_{2}(t) Q_{6}^{\frac{1}{2}}(t)\right\|_{H}^{2}+\left\|d_{1}(t) Q_{7}^{\frac{1}{2}}(t)\right\|_{H}^{2} .
\end{aligned}
$$

Applying Young's and/or Holder's inequalities to each term in the right hand side of (44) yields

$$
\begin{aligned}
T & \leq\left\|\lambda_{1}\left(a_{1}(t)+\gamma b_{1}(t)\right)^{\frac{1}{2}} X_{1}\right\|_{H}^{2}+\left\|\frac{1}{2 \lambda_{1}}\left(a_{1}(t)+\gamma b_{1}(t)\right)^{\frac{1}{2}} X_{2}\right\|_{H}^{2}+\left\|\lambda_{2}\left|a_{2}(t)\right|^{\frac{1}{2}} X_{1}^{2}\right\|_{H}^{2}+\left\|\frac{1}{2 \lambda_{2}}\left|a_{2}(t)\right|^{\frac{1}{2}} X_{2}\right\|_{H}^{2}+\left\|\frac{3^{\frac{1}{2}} \lambda_{3}^{\frac{2}{3}}}{2} a_{3}^{\frac{1}{3}}(t) X_{1}^{2}\right\|_{H}^{2} \\
& +\left\|\frac{1}{2 \lambda_{3}^{2}} a_{3}(t) X_{2}^{2}\right\|_{H}^{2}+\left\|\lambda_{4}\left|b_{2}(t)\right|^{\frac{1}{2}} X_{2}^{2}\right\|_{H}^{2}+\left\|\frac{1}{2 \lambda_{4}}\left|b_{2}(t)\right|^{\frac{1}{2}} X_{2}\right\|_{H}^{2}+\left\|\lambda_{5}\left|\gamma a_{2}(t)\right|^{\frac{1}{2}} X_{1}^{2}\right\|_{H}^{2}+\left\|\frac{\gamma^{\frac{1}{2}}}{2 \lambda_{5}}\left|a_{2}(t)\right|^{\frac{1}{2}} X_{1}\right\|_{H}^{2}+\left\|\lambda_{6}\left|\gamma b_{2}(t)\right|^{\frac{1}{2}} X_{1}\right\|_{H}^{2} \\
& +\left\|\frac{\gamma^{\frac{1}{2}}}{2 \lambda_{6}}\left|b_{2}(t)\right|^{\frac{1}{2}} X_{2}^{2}\right\|_{H}^{2}+\left\|\frac{3^{\frac{1}{2}} \gamma^{\frac{1}{2}} \lambda_{7}^{\frac{2}{3}}}{2} b_{3}^{\frac{1}{3}}(t) X_{2}^{2}\right\|_{H}^{2}+\left\|\frac{\gamma^{\frac{1}{2}}}{2 \lambda_{7}^{2}} b_{3}(t) X_{1}^{2}\right\|_{H}^{2}+\left\|\gamma^{\frac{1}{2}} \lambda_{8} X_{1}\right\|_{H}^{2}+\left\|\lambda_{9} X_{2}\right\|^{2}+\left\|\left(\frac{\gamma^{\frac{1}{2}}}{2 \lambda_{8}}+\frac{1}{2 \lambda_{9}}\right) f_{0}(t)\right\|_{H}^{2} \\
& +\left\|c_{2}(t) Q_{1}^{\frac{1}{2}}(t) X_{1}^{2}\right\|_{H}^{2}+\left\|c_{2}(t) Q_{2}^{\frac{1}{2}}(t) X_{1}\right\|_{H}^{2}+\left\|d_{2}(t) Q_{3}^{\frac{1}{2}}(t) X_{2}^{2}\right\|_{H}^{2}+\left\|d_{2}(t) Q_{4}^{\frac{1}{2}}(t) X_{2}\right\|_{H}^{2}+\left\|c_{1}(t) Q_{5}^{\frac{1}{2}}(t)\right\|_{H}^{2}+\left\|c_{2}(t) Q_{6}^{\frac{1}{2}}(t)\right\|_{H}^{2} \\
& +\left\|d_{1}(t) Q_{7}^{\frac{1}{2}}(t)\right\|_{H}^{2},
\end{aligned}
$$


where $\lambda_{i}, i=1, \ldots, 9$ are positive constants. Substituting (45) into (43) results in

$$
\begin{aligned}
\mathcal{L} U & \leq-\gamma E I\left\|\mathbb{D}^{2} X_{1}\right\|_{H}^{2}-\gamma P_{0}\left\|\mathbb{D} X_{1}\right\|_{H}^{2}-\frac{\gamma E a}{2}\left\|\left(\mathbb{D} X_{1}\right)^{2}\right\|_{H}^{2}-\left\|k_{11}(t) X_{1}\right\|_{H}^{2}-\left\|k_{12}(t) X_{1}^{2}\right\|_{H}^{2} \\
& -\left\|k_{21}(t) X_{2}\right\|_{H}^{2}-\left\|k_{22}(t) X_{2}^{2}\right\|_{H}^{2}+\varphi_{B}\left(\gamma X_{1}^{B L}+X_{2}^{B L}\right)+k_{0}(t),
\end{aligned}
$$

where $k_{11}(t), k_{12}(t), k_{21}(t)$, and $k_{22}(t)$ are defined as follows

$$
\begin{aligned}
& k_{11}(t)=\left(\gamma a_{1}(t)\right)^{\frac{1}{2}}-\lambda_{1}\left(a_{1}(t)+\gamma b_{1}(t)\right)^{\frac{1}{2}}-\frac{\gamma^{\frac{1}{2}}}{2 \lambda_{5}}\left|a_{2}(t)\right|^{\frac{1}{2}}-\lambda_{6}\left|\gamma b_{2}(t)\right|^{\frac{1}{2}}-\gamma^{\frac{1}{2}} \lambda_{8}-c_{2}(t) Q_{2}^{\frac{1}{2}}(t), \\
& k_{12}(t)=\left(\gamma a_{3}(t)\right)^{\frac{1}{2}}-\lambda_{2}\left|a_{2}(t)\right|^{\frac{1}{2}}-\frac{3^{\frac{1}{2}} \lambda_{3}^{\frac{2}{3}}}{2} a_{3}^{\frac{1}{3}}(t)-\lambda_{5}\left|\gamma a_{2}(t)\right|^{\frac{1}{2}}-\frac{\gamma^{\frac{1}{2}}}{2 \lambda_{7}^{2}} b_{3}(t)-c_{2}(t) Q_{1}^{\frac{1}{2}}(t), \\
& k_{21}(t)=b_{1}^{\frac{1}{2}}(t)-(\gamma \varrho a)^{\frac{1}{2}}-\frac{1}{2 \lambda_{1}}\left(a_{1}(t)+\gamma b_{1}(t)\right)^{\frac{1}{2}}-\frac{1}{2 \lambda_{2}}\left|a_{2}(t)\right|^{\frac{1}{2}}-\frac{1}{2 \lambda_{4}}\left|b_{2}(t)\right|^{\frac{1}{2}}-\lambda_{9}-d_{2}(t) Q_{4}^{\frac{1}{2}}(t), \\
& k_{22}(t)=b_{3}^{\frac{1}{2}}(t)-\frac{1}{2 \lambda_{3}^{2}} a_{3}(t)-\lambda_{4}\left|b_{2}(t)\right|^{\frac{1}{2}}-\frac{\gamma^{\frac{1}{2}}}{2 \lambda_{6}}\left|b_{2}(t)\right|^{\frac{1}{2}}-\frac{3^{\frac{1}{2}} \gamma^{\frac{1}{2}} \lambda_{7}^{\frac{2}{3}}}{2} b_{3}^{\frac{1}{3}}(t)-d_{2}(t) Q_{3}^{\frac{1}{2}}(t),
\end{aligned}
$$

and

$$
k_{0}(t)=\left\|\left(\frac{\gamma^{\frac{1}{2}}}{2 \lambda_{8}}+\frac{1}{2 \lambda_{9}}\right) f_{0}(t)\right\|_{H}^{2}+\left\|c_{1}(t) Q_{5}^{\frac{1}{2}}(t)\right\|_{H}^{2}+\left\|c_{2}(t) Q_{6}^{\frac{1}{2}}(t)\right\|_{H}^{2}+\left\|d_{1}(t) Q_{7}^{\frac{1}{2}}(t)\right\|_{H}^{2}
$$

We choose the $\gamma$ satisfying (39) and positive constants $\lambda_{i}, i=1, \ldots, 9$ such that $k_{11}(t), k_{12}(t), k_{21}(t)$ and $k_{22}(t)$ are strictly positive. This choice is always possible under Assumption 2.1.2 provided that we choose a sufficiently small $\gamma$.

From (46), we choose the boundary control $\varphi_{B}$ as a function of $X_{1}^{B L}$ and $X_{2}^{B L}$ as follows

$$
\varphi_{B}=-k_{B}\left(\gamma X_{1}^{B L}+X_{2}^{B L}\right),
$$

where $k_{B}$ is a positive constant. Substituting (49) into (46) yields

$$
\begin{aligned}
\mathcal{L} U & \leq-\gamma E I\left\|\mathbb{D}^{2} X_{1}\right\|_{H}^{2}-\gamma P_{0}\left\|\mathbb{D} X_{1}\right\|_{H}^{2}-\frac{\gamma E a}{2}\left\|\left(\mathbb{D} X_{1}\right)^{2}\right\|_{H}^{2}-\left\|k_{11}(t) X_{1}\right\|_{H}^{2}-\left\|k_{12}(t) X_{1}^{2}\right\|_{H}^{2}-\left\|k_{21}(t) X_{2}\right\|_{H}^{2} \\
& -\left\|k_{22}(t) X_{2}^{2}\right\|_{H}^{2}-k_{B}\left(\gamma X_{1}^{B L}+X_{2}^{B L}\right)^{2}+k_{0}(t) .
\end{aligned}
$$

The control design has been completed. The main results are summarized in the following theorem.

Theorem 4.1. Under Assumption 2.1, the boundary control $\varphi_{B}$ given in (49) solves Control Objective 2.1. In particular, the closed-loop system consisting of (35), (36), and (49) has a global unique variational solution, and this solution almost sure globally practically stable at the origin in the $\mathcal{H}$ norm.

Proof. See Appendix C.

\section{Simulations}

This section illustrate the effectiveness of the proposed boundary controller via some numerical simulations. The beam and distributed force parameters are given in Table 1. The membrane displacement is taken as

$$
\vartheta_{d}=0.5 \sin (0.1 t), \vartheta_{s}=\sum_{i=1}^{N_{\vartheta}} A_{\vartheta i} \vartheta_{\vartheta i} \dot{w}_{i},
$$

where $w_{i}$ is the standard Wiener process, the amplitude $A_{\vartheta i}$, wave number $k_{\vartheta i}$, and frequency $\vartheta_{\vartheta i}$ of the wave $i^{\text {th }}$ are given by

$$
\begin{aligned}
& \vartheta_{\vartheta i}=\vartheta_{m}+\frac{\vartheta_{m}-\vartheta_{M}}{N_{\vartheta}} i, S_{\vartheta i}=\frac{1.25}{4} \frac{\vartheta_{o}^{4}}{\vartheta_{\vartheta i}^{5}} H_{s \vartheta}^{2} e^{-1.25\left(\frac{\vartheta_{o}}{\vartheta_{\vartheta i}}\right)^{4}} \\
& A_{\vartheta i}=\sqrt{2 S_{\vartheta i} \frac{\vartheta_{m i}-\vartheta_{M i}}{N_{\vartheta}}}, 9.8 k_{\vartheta i} \tanh \left(k_{\vartheta i} L\right)=\vartheta_{\vartheta i}^{2} .
\end{aligned}
$$




\begin{tabular}{|l|c|c|}
\hline Variable & Value & Unit \\
\hline Length $L$ & 500 & $\mathrm{~m}$ \\
\hline Outer diameter $d_{o}$ & 0.1 & $\mathrm{~m}$ \\
\hline Inner diameter $d_{\text {in }}$ & 0.08 & $\mathrm{~m}$ \\
\hline Mass density $\varrho$ & 8200 & $\mathrm{~kg} / \mathrm{m}^{3}$ \\
\hline Young's modulus $E$ & $2 \times 10^{8}$ & $\mathrm{~kg} / \mathrm{m}^{2}$ \\
\hline Initial tension $P_{0}$ & $60 \times 10^{3}$ & $\mathrm{~N}$ \\
\hline Linear restoring coefficient $c_{1}(z, t)$ & 200 & $\mathrm{~N} / \mathrm{m}$ \\
\hline Nonlinear restoring coefficient $c_{2}(z, t)$ & 150 & $\mathrm{~N} / \mathrm{m}^{3}$ \\
\hline Linear damping coefficient $d_{1}(z, t)$ & 300 & $\mathrm{Ns} / \mathrm{m}$ \\
\hline Nonlinear damping coefficient $d_{2}(z, t)$ & 250 & $\mathrm{Ns} / \mathrm{m}^{3}$ \\
\hline
\end{tabular}

Table 1: Riser and distributed force parameters

In (52), the minimum and maximum wave frequencies are $\vartheta_{m}=0.1 \mathrm{rand} / \mathrm{s}, \vartheta_{M}=1 \mathrm{rand} / \mathrm{s}$; the two-parameter Bretschneider spectrum $S_{\vartheta i}$ is used with the significant wave height $H_{s \vartheta}=0.2 \mathrm{~m}$; the modal frequency is $\vartheta_{o}=\frac{2 \pi}{T_{\vartheta}}$ with the period $T_{\vartheta}=0.38 ; N_{\vartheta}=10$.

The fluid/air velocity is assumed to be

$$
v_{d}=5+0.5 \cos (0.5 t), \quad v_{s}=\sum_{i=1}^{N_{v}} A_{v i} v_{v i} \frac{\cosh \left(k_{v i} z\right)}{\sinh \left(k_{v i} L\right)} \dot{w}_{i}
$$

where the amplitude $A_{v i}$, wave number $k_{v i}$, and frequency $v_{v i}$ of the wave $i^{\text {th }}$ are given by

$$
\begin{aligned}
& v_{v i}=v_{m}+\frac{v_{m}-v_{M}}{N_{v}} i, S_{v i}=\frac{1.25}{4} \frac{v_{o}^{4}}{v_{v i}^{5}} H_{s v}^{2} e^{-1.25\left(\frac{v_{o}}{v_{v i}}\right)^{4}} \\
& A_{v i}=\sqrt{2 S_{v i} \frac{v_{m i}-v_{M i}}{N_{v}}}, 9.8 k_{v i} \tanh \left(k_{v i} L\right)=v_{v i}^{2},
\end{aligned}
$$

In (54), the minimum and maximum wave frequencies are $v_{m}=0.2 \mathrm{rand} / \mathrm{s}, v_{M}=2.5 \mathrm{rand} / \mathrm{s}$; the two-parameter Bretschneider spectrum $S_{v i}$ is used with the significant wave height $H_{s v}=4 m$; the modal frequency is $v_{o}=\frac{2 \pi}{T_{v}}$ with the period $T_{v}=7.8$; and $N_{v}=10$.

The initial conditions are taken as $t_{0}=0, u_{0}(z)=2 \sin \left(\frac{4 \pi}{L} z\right)$ and $u_{1}(z)=0$. The control gain $k_{B}$ and the constant $\gamma$ are chosen as $k_{B}=5$ and $\gamma=10^{-2}$. It is directly verified that Assumption 2.1 holds. We use a finite difference scheme, see Richtmyer and Morton (1967), to approximate the uncontrolled/controlled (continuous) system for the simulation purposes.

We run simulations without the proposed boundary controller, i.e. we set the control gain to zero $\left(k_{B}=0\right)$, and with the proposed boundary controller, i.e. $k_{B}=5$ and $\gamma=10^{-2}$. The length of simulation time for both cases is 500 seconds. The transverse displacement $u(z, t)$ for the uncontrolled and controlled cases are displayed in Figures 3 and 4, respectively. It is seen from these figures that the proposed boundary controller can reduce deflections of the riser transverse motion significantly, i.e. the displacement magnitudes are significantly reduced. This illustrates the effectiveness of the proposed boundary controller in the sense that it is able to drive the riser to the small neighborhood of its equilibrium position. It is noted that if one uses a deterministic simulation, the transverse motion $u(z, t)$ will be very large even go to infinity depending on how the stochastic terms saturated, see Remark 2.1.

\section{Conclusions}

Sufficient conditions have been derived to ensure global well-posedness and a.s. stability for a class of nonlinear SESs. The results were then applied to design boundary controllers achieving global well-posedness and a.s. practical stability of the flexible marine riser system under stochastic environmental loads. Future work is to apply the design method proposed in this paper to design boundary controllers for marine risers in three dimensional space and other flexible systems. 

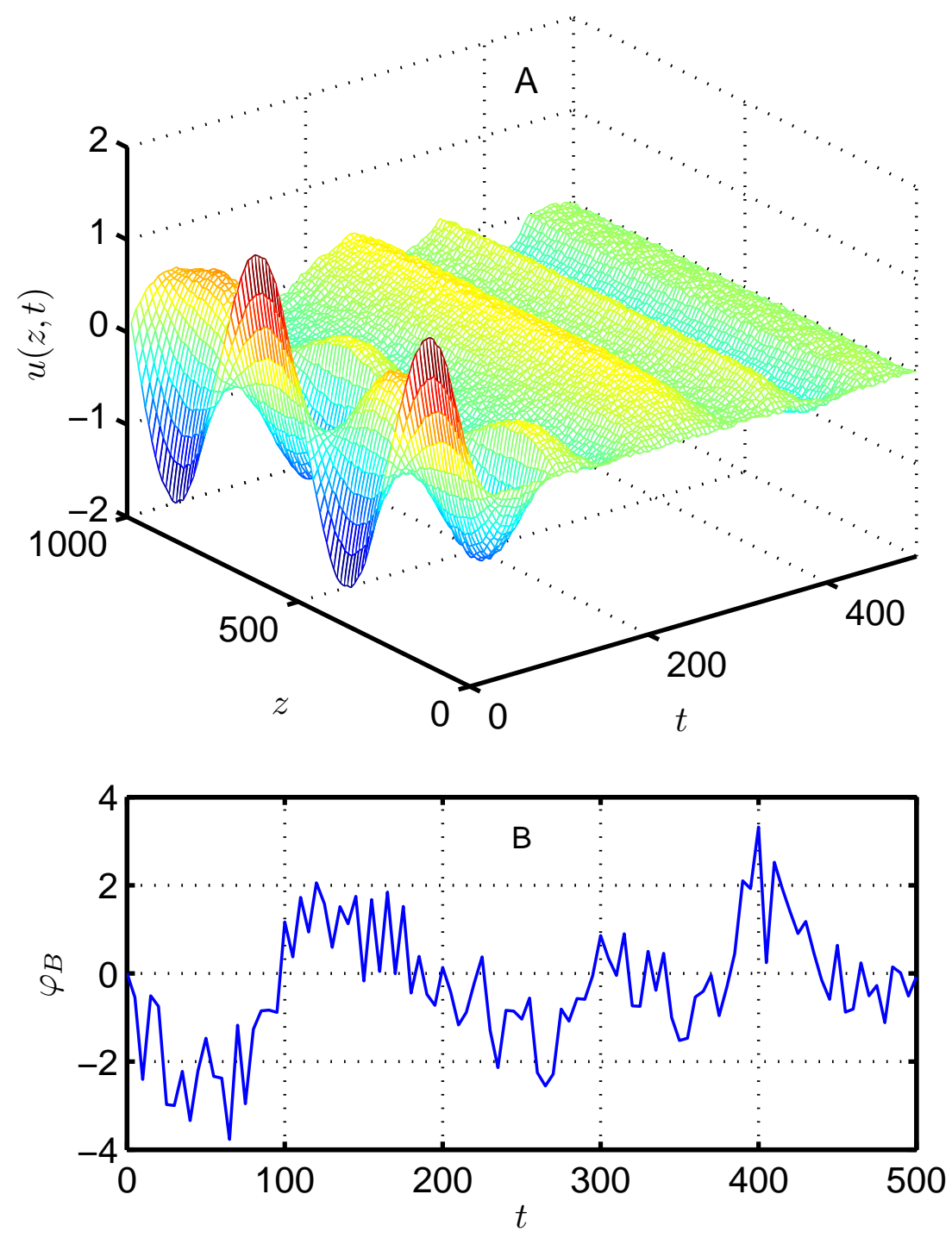

Figure 3: Simulation results with the proposed boundary control.

\section{Appendix A. Proof of Theorem 3.1}

To prepare for the proof of this theorem, we first prove existence and uniqueness of the solution for linear and semilinear SESs in the following two subsections.

Appendix A.1. Solution existence and uniqueness of linear SESs

We consider the following linear SES

$$
\begin{aligned}
& d \boldsymbol{X}_{1}=\boldsymbol{X}_{2} d t, \\
& d \boldsymbol{X}_{2}=\left[\boldsymbol{A}_{1 L}(t) \boldsymbol{X}_{1}+\boldsymbol{A}_{2 L}(t) \boldsymbol{X}_{2}+\boldsymbol{F}_{0}(t)\right] d t+d \boldsymbol{B}(t), \\
& \boldsymbol{X}_{1}\left(t_{0}\right)=\boldsymbol{X}_{10} \in V, \boldsymbol{X}_{2}\left(t_{0}\right)=\boldsymbol{X}_{20} \in H,
\end{aligned}
$$

where $\boldsymbol{X}_{1}$ and $\boldsymbol{X}_{2}$ are $n$-dimensional state vectors; $\boldsymbol{F}_{0}(t)$ is a predictable $H$-valued process, and $\boldsymbol{B}(t)$ is a continuous $L^{2}$-martingale in $H$ with local covariance operator $\boldsymbol{Q}(t)$ such that

$$
\mathbb{E}\left\{\int_{t_{0}}^{t}\left(\left\|\boldsymbol{F}_{0}(s)\right\|_{H}^{2}+\operatorname{Tr}(\boldsymbol{Q}(s))\right) d s\right\}<\infty
$$

for a.e. $(t, \omega) \in\left[t_{0}, \infty\right) \times \Omega$. Recalling that we defined $\mathcal{H}=V \times H$ and $\mathcal{V} \subset \mathcal{H}$, a subspace of $\mathcal{H}$, satisfies boundary conditions if applicable. The operator matrix $\boldsymbol{A}_{i L}(t), i=1,2$, where we use a short notation $\boldsymbol{A}_{i L}(t)$ for $\boldsymbol{A}_{i L}(t, \omega)$ with $t \in\left[t_{0}, \infty\right)$ and $\omega \in \Omega$, is supposed to satisfy the following assumption. 


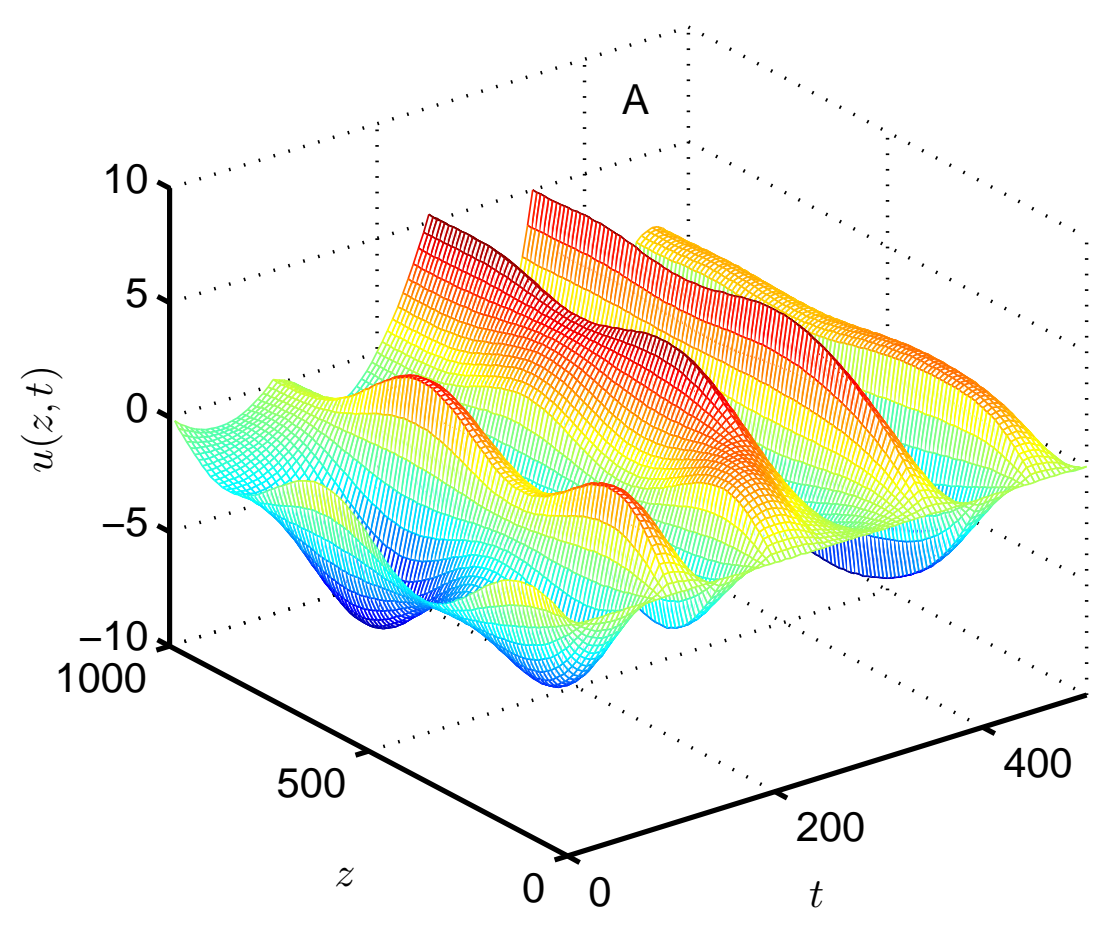

B

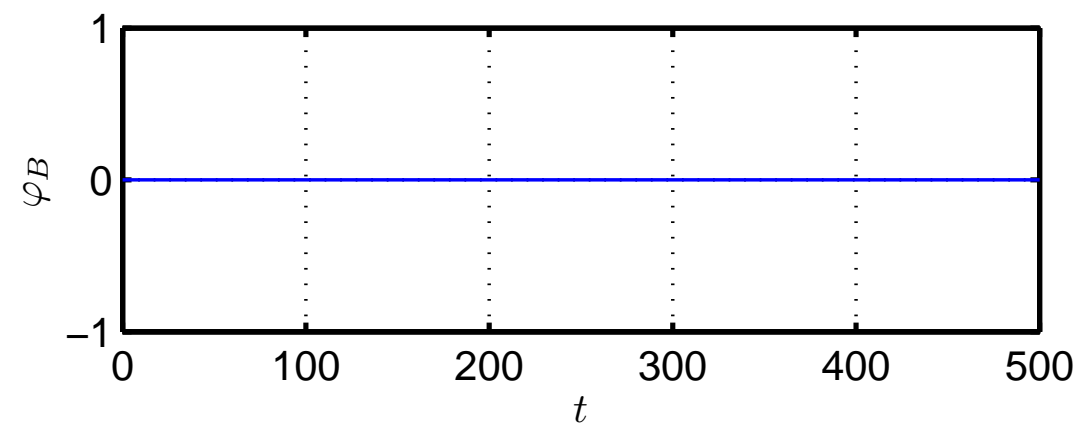

Figure 4: Simulation results without a boundary control.

\section{Assumption Appendix A.1.}

1) $\boldsymbol{A}_{i L}(t)$ are a continuous family of closed random linear operators with the domain $\mathcal{D}\left(\boldsymbol{A}_{i L}\right)$ dense in $H$ such that $\boldsymbol{A}_{i L}: V \rightarrow V^{*}$, and for any $\boldsymbol{X}_{1} \in V$ and $\boldsymbol{X}_{2} \in H, \boldsymbol{A}_{1 L}(t) \boldsymbol{X}_{1}$ and $\boldsymbol{A}_{2 L}(t) \boldsymbol{X}_{2}$ are adapted continuous $V^{*}$ - and $H$-valued processes, respectively.

2) For any $\boldsymbol{X}=\operatorname{col}\left(\boldsymbol{X}_{1}, \boldsymbol{X}_{2}\right) \in \mathcal{V}$, let us define

$$
U_{L}\left(\boldsymbol{X}_{1}, \boldsymbol{X}_{2}\right)=\frac{1}{2}\left\|\boldsymbol{X}_{1}\right\|_{V}^{2}+\frac{1}{2}\left\|\boldsymbol{X}_{2}\right\|_{H}^{2}
$$

There exists a nonnegative constant $\bar{b}_{1}$ such that the function $U_{L}\left(\boldsymbol{X}_{1}, \boldsymbol{X}_{2}\right)$ satisfies

$$
\mathbb{E}\left\{\sup _{t_{0} \leq \tau \leq t} U_{L}\left(\boldsymbol{X}_{1}(\tau), \boldsymbol{X}_{2}(\tau)\right)\right\} \leq \bar{b}_{1} \mathbb{E}\left\{\left\|\boldsymbol{X}_{10}\right\|_{V}^{2}+\left\|\boldsymbol{X}_{20}\right\|_{H}^{2}+\int_{t_{0}}^{t}\left(\left\|\boldsymbol{F}_{0}(s)\right\|_{H}^{2}+\operatorname{Tr}(\boldsymbol{Q}(s))\right) d s\right\}
$$

a.e. $(t, \omega) \in\left[t_{0}, \infty\right) \times \Omega$.

Existence and uniqueness of the solution of (A.1) is given in the following lemma.

Lemma Appendix A.1. Under Assumption Appendix A.1, suppose that $\boldsymbol{F}_{0}(t)$ is a predictable $H$-valued process, and $\boldsymbol{B}(t)$ is a continuous $L^{2}$-martingale in $H$ with local covariance operator $\boldsymbol{Q}(t)$ such that (A.2) holds. Then for each $\boldsymbol{X}_{10} \in V$ and $\boldsymbol{X}_{20} \in H$, the linear $S E S$ (A.1) has a unique variational solution with $\boldsymbol{X}_{1} \in L^{2}\left(\Omega ; C\left(\left[t_{0}, \infty\right) ; V\right)\right)$ and $\boldsymbol{X}_{2} \in L^{2}\left(\Omega ; C\left(\left[t_{0}, \infty\right) ; H\right)\right)$. 
Proof. Let $\phi_{k}, k=1,2, \ldots$ be orthogonal basis in $H$ and let $H_{n}=\operatorname{Span}\left\{\phi_{1}, \ldots, \phi_{n}\right\}$ such that $H_{n}$ is dense in $V$. Let $\boldsymbol{P}_{n}: V^{*} \rightarrow H_{n}$ defined by $\boldsymbol{P}_{n} \boldsymbol{v}:=\sum_{i=1}^{n}\left\langle\boldsymbol{y}, \phi_{i}\right\rangle_{V, V^{*}} \phi_{i}, \boldsymbol{y} \in V^{*}$. Then $\boldsymbol{P}_{n} \mid H$ is just the orthogonal projection onto $H_{n}$ in $H$, and note that $H_{n}$ is a finite dimensional space. Consider the following approximate system

$$
\begin{aligned}
& d \boldsymbol{X}_{1 n}=\boldsymbol{X}_{2 n} d t, \\
& d \boldsymbol{X}_{2 n}=\left[\boldsymbol{A}_{1 L}(t) \boldsymbol{X}_{1 n}+\boldsymbol{A}_{2 L}(t) \boldsymbol{X}_{2 n}+\boldsymbol{F}_{0}(t)\right] d t+d \boldsymbol{B}_{n}(t), \\
& \boldsymbol{X}_{1 n}\left(t_{0}\right)=\boldsymbol{X}_{10}, \boldsymbol{X}_{2 n}\left(t_{0}\right)=\boldsymbol{X}_{20},
\end{aligned}
$$

where $\left\{\boldsymbol{B}_{n}(t)\right\}$ is a sequence of continuous martingales in $H_{n}$ such that $\mathbb{E}\left\{\sup _{t_{0} \leq t \leq \infty}\left\|\boldsymbol{B}(t)-\boldsymbol{B}_{n}(t)\right\|_{H}^{2}\right\}=0$ as $n \rightarrow$ $\infty$. The approximate system (A.5) has a unique solution $\left(\boldsymbol{X}_{1 n}, \boldsymbol{X}_{2 n}\right) \in L^{2}\left(\Omega ; \mathcal{C}\left(\left[t_{0}, \infty\right) ; \mathcal{H}\right)\right)$ under Assumption Appendix A.1.

We now show convergence of the approximate sequences. Let $\left(\boldsymbol{X}_{1 n}, \boldsymbol{X}_{2 n}\right)$ and $\left(\boldsymbol{X}_{1 m}, \boldsymbol{X}_{2 m}\right)$ be solutions of (A.5). Define $\left(\boldsymbol{X}_{1 m n}, \boldsymbol{X}_{2 m n}\right)=\left(\boldsymbol{X}_{1 m}-\boldsymbol{X}_{1 n}, \boldsymbol{X}_{2 m}-\boldsymbol{X}_{2 n}\right)$, and $\boldsymbol{B}_{m n}(t)=\boldsymbol{B}_{m}(t)-\boldsymbol{B}_{n}(t)$. Then $\left(\boldsymbol{X}_{1 m n}, \boldsymbol{X}_{2 m n}\right)$ satisfies the linear SES:

$$
\begin{aligned}
& d \boldsymbol{X}_{1 m n}=\boldsymbol{X}_{2 m n} d t, \\
& d \boldsymbol{X}_{2 m n}=\left[\boldsymbol{A}_{1 L}(t) \boldsymbol{X}_{1 m n}+\boldsymbol{A}_{2 L}(t) \boldsymbol{X}_{2 m n}\right] d t+d \boldsymbol{B}_{m n}(t), \\
& \boldsymbol{X}_{1 m n}\left(t_{0}\right)=0, \boldsymbol{X}_{2 m n}\left(t_{0}\right)=0 .
\end{aligned}
$$

Applying the condition (A.4) to (A.6) yields

$$
\mathbb{E}\left\{\sup _{t_{0} \leq \tau \leq t}\left(\left\|\boldsymbol{X}_{2 m n}(\tau)\right\|_{H}^{2}+\left\|\boldsymbol{X}_{1 m n}(\tau)\right\|_{V}^{2}\right\} \leq C \mathbb{E}\left\{\int_{t_{0}}^{t} \operatorname{Tr}\left(\boldsymbol{Q}_{m n}(s)\right) d s\right\} \rightarrow 0\right.
$$

as $(m, n) \rightarrow \infty$, where $C$ is a nonnegative constant. Thus, $\left\{\left(\boldsymbol{X}_{1 n}, \boldsymbol{X}_{2 n}\right)\right\}$ is a Cauchy sequence in $\boldsymbol{S}$, which is the Banach space of adapted continuous processes in the space $L^{2}\left(\Omega ; C\left(\left[t_{0}, \infty\right) ; \mathcal{H}\right)\right)$ with the norm defined by $\mathbb{E} \sup _{\left.t_{0} \leq t \leq \infty\right)}\left(\left\|\boldsymbol{X}_{1 n}\right\|_{V}^{2}+\left\|\boldsymbol{X}_{2 n}\right\|_{H}^{2}\right)^{1 / 2}$.

Next, we show that $\left(\boldsymbol{X}_{1 n}, \boldsymbol{X}_{2 n}\right)$ converges to the strong solution of (A.1). For any $\boldsymbol{\phi} \in H$ and $\psi \in V$, applying the Itô formula to $\left\langle\boldsymbol{X}_{1 n}, \boldsymbol{\phi}\right\rangle_{H}$ and $\left\langle\boldsymbol{X}_{2 n}, \psi\right\rangle_{H}$ along the solutions of (A.5) gives

$$
\begin{aligned}
\left\langle\boldsymbol{X}_{1 n}(t), \boldsymbol{\phi}\right\rangle_{H} & =\left\langle\boldsymbol{X}_{10}, \boldsymbol{\phi}\right\rangle_{H}+\int_{0}^{t}\left\langle\boldsymbol{X}_{2 n}(s), \boldsymbol{\phi}\right\rangle_{H} d s, \\
\left\langle\boldsymbol{X}_{2 n}(t), \boldsymbol{\psi}\right\rangle_{H} & =\left\langle\boldsymbol{X}_{20}, \boldsymbol{\psi}\right\rangle_{H}+\left\langle\boldsymbol{B}_{n}(t), \boldsymbol{\psi}\right\rangle_{H}+\int_{0}^{t}\left\langle\boldsymbol{A}_{1 L}(s) \boldsymbol{X}_{1 n}(s)+\boldsymbol{A}_{2 L}(s) \boldsymbol{X}_{2 n}(s)+\boldsymbol{F}_{0}(s), \boldsymbol{\psi}\right\rangle_{V, V^{*}} d s .
\end{aligned}
$$

Taking the termwise limit of (A.8) yields the desired result that $\left(\boldsymbol{X}_{1}(t), \boldsymbol{X}_{2}(t)\right)$ is a strong or variational solution of (A.1). Proof of uniqueness is fairly straightforward by carrying similar calculations as above.

Appendix A.2. Solution existence and uniqueness of semilinear SESS

We consider the following semilinear SES

$$
\begin{aligned}
& d \boldsymbol{X}_{1}=\boldsymbol{X}_{2} d t, \\
& d \boldsymbol{X}_{2}=\left[\boldsymbol{A}_{1 L}(t) \boldsymbol{X}_{1}+\boldsymbol{A}_{2 L}(t) \boldsymbol{X}_{2}+\boldsymbol{F}_{L}\left(\boldsymbol{X}_{1}, \boldsymbol{X}_{2}, t\right)\right] d t+\boldsymbol{G}_{L}\left(\boldsymbol{X}_{1}, \boldsymbol{X}_{2}, t\right) d \boldsymbol{W}(t), \\
& \boldsymbol{X}_{1}\left(t_{0}\right)=\boldsymbol{X}_{10} \in V, \boldsymbol{X}_{2}\left(t_{0}\right)=\boldsymbol{X}_{20} \in H,
\end{aligned}
$$

where $\boldsymbol{F}_{L}: \mathcal{H} \times\left[t_{0}, T\right] \rightarrow V^{*}$ and $\boldsymbol{G}_{L}: \mathcal{H} \times\left[t_{0}, T\right] \rightarrow \mathscr{L}_{2}\left(K_{0}, \mathcal{H}\right)$ are Borel measurable, $\boldsymbol{W}$ is a $Q$-Wiener process, the operator matrix $\boldsymbol{A}_{i L}(t), i=1,2$ satisfy Assumption Appendix A.1, and other notations are defined in Subsection Appendix A.1 as for the system (A.1).

We now impose the following global linear growth and Lipschitz conditions on the nonlinear term $\boldsymbol{F}_{L}\left(\boldsymbol{X}_{1}, \boldsymbol{X}_{2}, t\right)$ and $\boldsymbol{G}_{L}\left(\boldsymbol{X}_{1}, \boldsymbol{X}_{2}, t\right)$.

\section{Assumption Appendix A.2.}

1) For any $\boldsymbol{u} \in V, \boldsymbol{v} \in H$, there exist nonnegative constants $\bar{b}_{2}$ and $\bar{b}_{3}$ such that

$$
\begin{aligned}
& \mathbb{E}\left\{\int_{t_{0}}^{T}\left(\left\|\boldsymbol{F}_{L}(0,0, t)\right\|_{H}^{2}+\left\|\boldsymbol{G}_{L}(0,0, t)\right\|_{\mathscr{L}_{2}^{0}}^{2}\right) d t\right\} \leq \bar{b}_{2}, \\
& \left\|\tilde{\boldsymbol{F}}_{L}(\boldsymbol{u}, \boldsymbol{v}, t)\right\|_{H}^{2}+\left\|\tilde{\boldsymbol{G}}_{L}(\boldsymbol{u}, \boldsymbol{v}, t)\right\|_{\mathscr{L}_{2}^{0}}^{2} \leq \bar{b}_{3}\left(1+\|\boldsymbol{u}\|_{V}^{2}+\|\boldsymbol{v}\|_{H}^{2}\right), \text { a.e. }(t, \omega) \in \Omega_{T},
\end{aligned}
$$

where $\tilde{\boldsymbol{F}}_{L}(\boldsymbol{u}, \boldsymbol{v}, t)=\boldsymbol{F}_{L}(\boldsymbol{u}, \boldsymbol{v}, t)-\boldsymbol{F}_{L}(0,0, t), \tilde{\boldsymbol{G}}_{L}(\boldsymbol{u}, \boldsymbol{v}, t)=\boldsymbol{G}_{L}(\boldsymbol{u}, \boldsymbol{v}, t)-\boldsymbol{G}_{L}(0,0, t)$, and $\Omega_{T}:=\left[t_{0}, \infty\right) \times \Omega$. 
2) For any $\boldsymbol{u}_{1}, \boldsymbol{u}_{2} \in V$ and $\boldsymbol{v}_{1}, \boldsymbol{v}_{2} \in H$, there exists a nonnegative constant $\bar{b}_{4}$ such that

$$
\begin{gathered}
\left\|\boldsymbol{F}_{L}\left(\boldsymbol{u}_{1}, \boldsymbol{v}_{1}, t\right)-\boldsymbol{F}_{L}\left(\boldsymbol{u}_{2}, \boldsymbol{v}_{2}, t\right)\right\|_{H}^{2}+\left\|\boldsymbol{G}_{L}\left(\boldsymbol{u}_{1}, \boldsymbol{v}_{1}, t\right)-\boldsymbol{G}_{L}\left(\boldsymbol{u}_{2}, \boldsymbol{v}_{2}, t\right)\right\|_{\mathscr{L}_{2}^{0}}^{2} \\
\quad \leq \bar{b}_{4}\left(\left\|\boldsymbol{u}_{1}-\boldsymbol{u}_{2}\right\|_{V}^{2}+\left\|\boldsymbol{v}_{1}-\boldsymbol{v}_{2}\right\|_{H}^{2}\right), \text { a.e. }(t, \omega) \in \Omega_{T} .
\end{gathered}
$$

Existence and uniqueness of the solution of (A.9) is given in the following lemma.

Lemma Appendix A.2. Under Assumptions Appendix A.1 and Appendix A.2, for each $\boldsymbol{X}_{10} \in V$ and $\boldsymbol{X}_{20} \in H$, the semilinear SES (A.9) has a unique variational solution with $\boldsymbol{X}_{1} \in L^{2}\left(\Omega ; C\left(\left[t_{0}, T\right] ; V\right)\right)$ and $\boldsymbol{X}_{2} \in L^{2}\left(\Omega ; C\left(\left[t_{0}, T\right] ; H\right)\right)$.

Proof. We prove Lemma Appendix A.2 by using the contraction mapping principle. As such, given $\boldsymbol{X}_{1} \in$ $L^{2}\left(\Omega ; C\left(\left[t_{0}, T\right] ; V\right)\right)$ and $\boldsymbol{X}_{2} \in L^{2}\left(\Omega ; C\left(\left[t_{0}, T\right] ; H\right)\right)$, let us consider the following linear SES

$$
\begin{aligned}
& d \boldsymbol{\mu}_{1}=\boldsymbol{\mu}_{2} d t, \\
& d \boldsymbol{\mu}_{2}=\left[\boldsymbol{A}_{1 L}(t) \boldsymbol{\mu}_{1}+\boldsymbol{A}_{2 L}(t) \boldsymbol{\mu}_{2}+\tilde{\boldsymbol{F}}_{L}\left(\boldsymbol{X}_{1}, \boldsymbol{X}_{2}, t\right)\right] d t+\tilde{\boldsymbol{G}}_{L}\left(\boldsymbol{X}_{1}, \boldsymbol{X}_{2}, t\right) d \boldsymbol{W}(t), \\
& \boldsymbol{\mu}_{1}\left(t_{0}\right)=\boldsymbol{X}_{10}, \boldsymbol{\mu}_{2}\left(t_{0}\right)=\boldsymbol{X}_{20} .
\end{aligned}
$$

Define $\boldsymbol{F}_{0}(t)=\tilde{\boldsymbol{F}}_{L}\left(\boldsymbol{X}_{1}, \boldsymbol{X}_{2}, t\right)$ and $\boldsymbol{B}(t)=\int_{t_{0}}^{t} \tilde{\boldsymbol{G}}_{L}\left(\boldsymbol{X}_{1}(s), \boldsymbol{X}_{2}(s), s\right) d \boldsymbol{W}(s)$ with local covariance operator $\boldsymbol{Q}(t)$. Then by the condition (A.10), we have

$$
\begin{aligned}
\mathbb{E}\left\{\int_{t_{0}}^{T}\left(\left\|\boldsymbol{F}_{0}(s)\right\|_{H}^{2}+\operatorname{Tr}(\boldsymbol{Q}(s))\right) d t\right\} & =\mathbb{E}\left\{\int_{t_{0}}^{T}\left(\left\|\tilde{\boldsymbol{F}}_{L}\left(\boldsymbol{X}_{1}, \boldsymbol{X}_{2}, t\right)\right\|_{H}^{2}+\left\|\tilde{\boldsymbol{G}}_{L}\left(\boldsymbol{X}_{1}, \boldsymbol{X}_{2}, t\right)\right\|_{\mathscr{L}_{2}^{0}}^{2}\right) d t\right\} \\
& \leq \bar{b}_{3}\left(T-t_{0}\right)\left(1+\mathbb{E}\left\{\sup _{t_{0} \leq t \leq T}\left(\left\|\boldsymbol{X}_{1}\right\|_{V}^{2}+\left\|\boldsymbol{X}_{2}\right\|_{H}^{2}\right)\right\}\right) .
\end{aligned}
$$

Thus, for $\left(\boldsymbol{X}_{1}, \boldsymbol{X}_{2}\right) \in L^{2}\left(\Omega ; C\left(\left[t_{0}, T\right] ; V \times H\right)\right)$, applying Lemma Appendix A.1 to (A.12) shows that the system (A.12) has a unique solution $\left(\boldsymbol{\mu}_{1}, \boldsymbol{\mu}_{2}\right)=\boldsymbol{\Gamma}\left(\boldsymbol{X}_{1}, \boldsymbol{X}_{2}\right) \in \boldsymbol{S}_{T}$ and that the solution map $\boldsymbol{\Gamma}: \boldsymbol{S}_{T} \rightarrow \boldsymbol{S}_{T}$ is well defined.

We now show that $\boldsymbol{\Gamma}$ is a contraction for small $T$. As such, let $\boldsymbol{X}_{1}, \hat{\boldsymbol{X}}_{1} \in L^{2}\left(\Omega ; C\left(\left[t_{0}, T\right] ; V\right)\right.$ and $\boldsymbol{X}_{2}, \hat{\boldsymbol{X}}_{2} \in$ $L^{2}\left(\Omega ; C\left(\left[t_{0}, T\right] ; H\right)\right.$. Let $\left(\hat{\boldsymbol{\mu}}_{1}, \hat{\boldsymbol{\mu}}_{2}\right)=\boldsymbol{\Gamma}\left(\hat{\boldsymbol{X}}_{1}, \hat{\boldsymbol{X}}_{2}\right)$. Define $\left.\tilde{\boldsymbol{\mu}}_{1}=\boldsymbol{\mu}_{1}-\hat{\boldsymbol{\mu}}_{1}\right)$ and $\left.\tilde{\boldsymbol{\mu}}_{2}=\boldsymbol{\mu}_{2}-\hat{\boldsymbol{\mu}}_{2}\right)$. Then $\tilde{\boldsymbol{\mu}}_{1}$ and $\tilde{\boldsymbol{\mu}}_{2}$ satisfy the following system

$$
\begin{aligned}
& d \tilde{\boldsymbol{\mu}}_{1}=\tilde{\boldsymbol{\mu}}_{2} d t, \\
& d \tilde{\boldsymbol{\mu}}_{2}=\left[\boldsymbol{A}_{1 L}(t) \tilde{\boldsymbol{\mu}}_{1}+\boldsymbol{A}_{2 L}(t) \tilde{\boldsymbol{\mu}}_{2}\left(\tilde{\boldsymbol{F}}_{L}\left(\boldsymbol{X}_{1}, \boldsymbol{X}_{2}, t\right)-\tilde{\boldsymbol{F}}_{L}\left(\hat{\boldsymbol{X}}_{1}, \hat{\boldsymbol{X}}_{2}, t\right)\right)\right] d t+\left[\tilde{\boldsymbol{G}}_{L}\left(\boldsymbol{X}_{1}, \boldsymbol{X}_{2}, t\right)-\tilde{\boldsymbol{G}}_{L}\left(\hat{\boldsymbol{X}}_{1}, \hat{\boldsymbol{X}}_{2}, t\right)\right] d \boldsymbol{W}(t), \\
& \tilde{\boldsymbol{\mu}}_{1}\left(t_{0}\right)=0, \tilde{\boldsymbol{\mu}}_{2}\left(t_{0}\right)=0 .
\end{aligned}
$$

Applying the conditions (A.4) and (A.11) to (A.14) results in

$$
\begin{aligned}
\mathbb{E}\left\{\sup _{t_{0} \leq t \leq T}\left(\left\|\tilde{\boldsymbol{\mu}}_{1}\right\|_{V}^{2}+\left\|\tilde{\boldsymbol{\mu}}_{2}\right\|_{H}^{2}\right)\right\} & \leq C \mathbb{E}\left\{\int_{t_{0}}^{T}\left(\left\|\tilde{\boldsymbol{F}}_{L}\left(\boldsymbol{X}_{1}, \boldsymbol{X}_{2}, t\right)-\tilde{\boldsymbol{F}}_{L}\left(\hat{\boldsymbol{X}}_{1}, \hat{\boldsymbol{X}}_{2}, t\right)\right\|_{H}^{2}+\left\|\tilde{\boldsymbol{G}}_{L}\left(\boldsymbol{X}_{1}, \boldsymbol{X}_{2}, t\right)-\tilde{\boldsymbol{G}}_{L}\left(\hat{\boldsymbol{X}}_{1}, \hat{\boldsymbol{X}}_{2}, t\right)\right\|_{\mathscr{L}_{2}^{0}}^{2}\right) d t\right\} \\
& \leq C\left(T-t_{0}\right) \mathbb{E}\left\{\left\|\boldsymbol{X}_{1}-\hat{\boldsymbol{X}}_{1}\right\|_{V}^{2}+\left\|\boldsymbol{X}_{2}-\hat{\boldsymbol{X}}_{2}\right\|_{H}^{2}\right\},
\end{aligned}
$$

where $C$ is a positive constant, and we have used $\tilde{\boldsymbol{F}}_{L}(\bullet)-\tilde{\boldsymbol{F}}_{L}(\hat{\bullet})=\boldsymbol{F}_{L}(\bullet)-\boldsymbol{F}_{L}(\hat{\bullet})$ and $\tilde{\boldsymbol{G}}_{L}(\bullet)-\tilde{\boldsymbol{G}}_{L}(\boldsymbol{\bullet})=\boldsymbol{G}_{L}(\bullet)-\boldsymbol{G}_{L}(\hat{\bullet})$. Thus, $\boldsymbol{\Gamma}: \boldsymbol{S}_{T} \rightarrow \boldsymbol{S}_{T}$ is a contraction mapping for small $T$, and the unique fixed point $\left(\boldsymbol{X}_{1}, \boldsymbol{X}_{2}\right)$ is the solution of the system (A.9), which can be continued for any $T>t_{0}$.

\section{Appendix A.3. Proof of Theorem 3.1}

Let $k_{0}$ be the boundedness of the initial data, i.e., $\left\|\boldsymbol{X}_{0}\right\|_{\mathcal{H}} \leq k_{0}$. For any integer $k>k_{0}$, let us define

$$
\begin{aligned}
& \boldsymbol{F}_{k}(\boldsymbol{X}, t)=\boldsymbol{F}\left(\frac{\|\boldsymbol{X}\|_{\mathcal{H}} \wedge k}{\|\boldsymbol{X}\|_{\mathcal{H}}} \boldsymbol{X}, t\right), \\
& \boldsymbol{G}_{k}(\boldsymbol{X}, t)=\boldsymbol{G}\left(\frac{\|\boldsymbol{X}\|_{\mathcal{H}} \wedge k}{\|\boldsymbol{X}\|_{\mathcal{H}}} \boldsymbol{X}, t\right),
\end{aligned}
$$

where we let $\frac{\|\boldsymbol{X}\|_{\mathcal{H}} \wedge k}{\|\boldsymbol{X}\|_{\mathcal{H}}} \boldsymbol{X}=0$ if $\boldsymbol{X}=0$. Then, it is clear that Assumption 3.2 holds for all $\boldsymbol{X} \in \mathcal{H}$ with $\|\boldsymbol{X}\|_{\mathcal{H}} \leq$ $\varepsilon, \varepsilon>0$. Thus, Lemma Appendix A.2 ensures existence and uniqueness of the variational solution $\boldsymbol{X}_{k}(t)$ in $L^{2}\left(\Omega ; C\left(\left[t_{0}, T\right] ; \mathcal{H}\right)\right)$ for arbitrarily $T>0$ to the following SES:

$$
d \boldsymbol{X}_{k}=\boldsymbol{F}_{k}\left(\boldsymbol{X}_{k}, t\right) d t+\boldsymbol{G}_{k}\left(\boldsymbol{X}_{k}, t\right) d \boldsymbol{W}(t)
$$


for any $\boldsymbol{X}_{k}\left(t_{0}\right)=\boldsymbol{X}_{0} \in H$.

Define a stopping time

$$
s_{k}=\inf \left\{t \geq t_{0}:\left\|\boldsymbol{X}_{k}(t)\right\|_{\mathcal{H}}>k\right\},
$$

where we let $\inf \emptyset=\infty$ as usual. It is obvious that for any $\sigma<s_{k}$ we have $\left\|\boldsymbol{X}_{k}(\sigma)\right\|_{\mathcal{H}} \leq k$. From the definition of $\boldsymbol{F}_{k}(\boldsymbol{X}, t)$ and $\boldsymbol{G}_{k}(\boldsymbol{X}, t)$ in (A.16), it is seen that

$$
\begin{aligned}
& \boldsymbol{F}_{k+1}\left(\boldsymbol{X}_{k}(\sigma), t\right)=\boldsymbol{F}_{k}\left(\boldsymbol{X}_{k}(\sigma), t\right), \\
& \boldsymbol{G}_{k+1}\left(\boldsymbol{X}_{k}(\sigma), t\right)=\boldsymbol{G}_{k}\left(\boldsymbol{X}_{k}(\sigma), t\right),
\end{aligned}
$$

for all $t_{0} \leq \sigma \leq s_{k}$. Using (A.19), we have by the Itô formula:

$$
\begin{aligned}
\boldsymbol{X}_{k}\left(t \wedge s_{k}\right) & =\boldsymbol{X}_{0}+\int_{t_{0}}^{t \wedge s_{k}}\left(\boldsymbol{F}_{k}\left(\boldsymbol{X}_{k}(\sigma), \sigma\right) d \sigma+\boldsymbol{G}_{k}\left(\boldsymbol{X}_{k}(\sigma), \sigma\right) d \boldsymbol{W}(\sigma)\right) \\
& =\boldsymbol{X}_{0}+\int_{t_{0}}^{t \wedge s_{k}}\left(\boldsymbol{F}_{k+1}\left(\boldsymbol{X}_{k}(\sigma), \sigma\right) d \sigma+\boldsymbol{G}_{k+1}\left(\boldsymbol{X}_{k}(\sigma), \sigma\right) d \boldsymbol{W}(\sigma)\right),
\end{aligned}
$$

which implies that

$$
\boldsymbol{X}_{k+1}(t)=\boldsymbol{X}_{k}(t), \quad \forall t_{0} \leq t \leq s_{k},
$$

and that $s_{k}$ is increasing in $k$. This means that we can define $s=\lim _{k \rightarrow \infty} s_{k}$. From (A.21), we also have

$$
\boldsymbol{X}(t)=\boldsymbol{X}_{k}(t), \quad \forall t_{0} \leq t<s_{k}
$$

This together with (A.19) means that $\boldsymbol{X}(t)$ is the unique strong solution of (16) for $t \in\left[t_{0}, s_{k}\right)$. We now need to show that $\mathbb{P}(s=\infty)=1$. Applying the Itô formula to $U\left(\boldsymbol{X}_{k}, t\right)$ with $\boldsymbol{X}_{k}$ being the strong solution of (A.17) results in

$$
U\left(\boldsymbol{X}_{k}\left(t \wedge s_{k}\right), t\right)=U\left(\boldsymbol{X}_{0}, t_{0}\right)+\int_{t_{0}}^{t \wedge s_{k}} \mathcal{L}_{k} U\left(\boldsymbol{X}_{k}(\sigma), \sigma\right) d \sigma+\int_{t_{0}}^{t \wedge s_{k}}\left\langle U_{\boldsymbol{X}_{k}}\left(\boldsymbol{X}_{k}(\sigma), \sigma\right), \boldsymbol{G}_{k}\left(\boldsymbol{X}_{k}(\sigma), \sigma\right) d \boldsymbol{W}(\sigma)\right\rangle_{\mathcal{H}},
$$

where

$$
\begin{aligned}
\mathcal{L}_{k} U\left(\boldsymbol{X}_{k}(\sigma), \sigma\right) & =U_{\sigma}\left(\boldsymbol{X}_{k}(\sigma), \sigma\right)+\left\langle\boldsymbol{F}_{k}\left(\boldsymbol{X}_{k}(\sigma), \sigma\right), U_{\boldsymbol{X}_{k}}\left(\boldsymbol{X}_{k}(\sigma), \sigma\right)\right\rangle_{\mathcal{H}} \\
& +\frac{1}{2} \operatorname{Tr}\left(U_{\boldsymbol{X}_{k} \boldsymbol{X}_{k}}(\boldsymbol{X}(\sigma), \sigma) \boldsymbol{G}_{k}\left(\boldsymbol{X}_{k}(\sigma), \sigma\right) \boldsymbol{Q \boldsymbol { G } _ { k } ^ { \star }}\left(\boldsymbol{X}_{k}, \sigma\right)\right) .
\end{aligned}
$$

By definition of $\boldsymbol{F}_{k}$ and $\boldsymbol{G}_{k}$ in (A.16), we have

$$
\mathcal{L}_{k} U\left(\boldsymbol{X}_{k}(\sigma), \sigma\right)=\mathcal{L} U\left(\boldsymbol{X}_{k}(\sigma), \sigma\right), \quad \forall \sigma<t \wedge s_{k},
$$

where

$$
\begin{aligned}
\mathcal{L} U\left(\boldsymbol{X}_{k}(\sigma), \sigma\right) & =U_{\sigma}\left(\boldsymbol{X}_{k}(\sigma), \sigma\right)+\left\langle\boldsymbol{F}\left(\boldsymbol{X}_{k}(\sigma), \sigma\right), U_{\boldsymbol{X}_{k}}\left(\boldsymbol{X}_{k}(\sigma), \sigma\right)\right\rangle_{\mathcal{H}} \\
& +\frac{1}{2} \operatorname{Tr}\left(U_{\boldsymbol{X}_{k} \boldsymbol{X}_{k}}(\boldsymbol{X}(\sigma), \sigma) \boldsymbol{G}\left(\boldsymbol{X}_{k}(\sigma), \sigma\right) \boldsymbol{Q} \boldsymbol{G}^{\star}\left(\boldsymbol{X}_{k}, \sigma\right)\right) .
\end{aligned}
$$

Moreover, since $\int_{t_{0}}^{t \wedge s_{k}}\left\langle U_{\boldsymbol{X}_{k}}\left(\boldsymbol{X}_{k}(\sigma), \sigma\right), \boldsymbol{G}_{k}\left(\boldsymbol{X}_{k}(\sigma), \sigma\right) d \boldsymbol{W}(\sigma)\right\rangle_{\mathcal{H}}$ is a martingale, we have

$$
\mathbb{E}\left\{\int_{t_{0}}^{t \wedge s_{k}}\left\langle U_{\boldsymbol{X}_{k}}\left(\boldsymbol{X}_{k}(\sigma), \sigma\right), \boldsymbol{G}_{k}\left(\boldsymbol{X}_{k}(\sigma), \sigma\right) d \boldsymbol{W}(\sigma)\right\rangle_{\mathcal{H}}\right\}=0,
$$

which also implies that

$$
\mathbb{E}\left\{\int_{t_{0}}^{t \wedge s_{k}}\left\langle U_{\boldsymbol{X}_{k}}\left(\boldsymbol{X}_{k}(\sigma), \sigma\right), \boldsymbol{G}\left(\boldsymbol{X}_{k}(\sigma), \sigma\right) d \boldsymbol{W}(\sigma)\right\rangle_{\mathcal{H}}\right\}=0 .
$$

Taking expectation both sides of (A.23) and using (A.28) and (27) give

$$
\begin{aligned}
\mathbb{E}\left\{U\left(\boldsymbol{X}_{k}\left(t \wedge s_{k}\right), t\right)\right\} & =\mathbb{E}\left\{U\left(\boldsymbol{X}_{0}, t_{0}\right)\right\}+\mathbb{E}\left\{\int_{t_{0}}^{t \wedge s_{k}} \mathcal{L} U\left(\boldsymbol{X}_{k}(\sigma), \sigma\right) d \sigma\right\} \\
& \leq \mathbb{E}\left\{U\left(\boldsymbol{X}_{0}, t_{0}\right)\right\}+c \mathbb{E}\left\{\int_{t_{0}}^{t \wedge s_{k}}\left(1+U\left(\boldsymbol{X}_{k}(\sigma), \sigma\right)\right) d \sigma\right\} \\
& \leq \mathbb{E}\left\{U\left(\boldsymbol{X}_{0}, t_{0}\right)\right\}+c \mathbb{E}\left\{\int_{t_{0}}^{t}\left(1+U\left(\boldsymbol{X}_{k}\left(\sigma \wedge s_{k}\right), \sigma\right)\right) d \sigma\right\} .
\end{aligned}
$$


Applying the Gronwall inequality, see Adams and Fournier (2003), to (A.29) yields

$$
\mathbb{E}\left\{U\left(\boldsymbol{X}_{k}\left(t \wedge s_{k}\right), t\right)\right\} \leq\left(c\left(t-t_{0}\right)+\mathbb{E}\left\{U\left(\boldsymbol{X}_{0}, t_{0}\right)\right\}\right) e^{c\left(t-t_{0}\right)},
$$

which further gives

$$
\begin{aligned}
\inf _{\|\boldsymbol{X}\|_{\mathcal{H}} \geq k} U(\boldsymbol{X}, t) \mathbb{P}\left(s_{k} \leq t\right) & \leq \mathbb{E}\left\{U\left(\boldsymbol{X}_{k}\left(s_{k}\right), t\right) \mathscr{I}_{s_{k} \leq t}\right\} \\
& \leq\left(c\left(t-t_{0}\right)+\mathbb{E}\left\{U\left(\boldsymbol{X}_{0}, t_{0}\right)\right\}\right) e^{c\left(t-t_{0}\right)}
\end{aligned}
$$

Therefore

$$
\mathbb{P}\left(s_{k} \leq t\right) \leq \frac{\left(c\left(t-t_{0}\right)+\mathbb{E}\left\{U\left(\boldsymbol{X}_{0}, t_{0}\right)\right\}\right) e^{c\left(t-t_{0}\right)}}{\inf _{\|\boldsymbol{X}\|_{\mathcal{H}} \geq k} U(\boldsymbol{X}, t)} .
$$

Letting $k$ tend to infinity and using (28) result in $\mathbb{P}\left(s_{k} \leq t\right)=0$. Since $t$ is arbitrary, we have $\mathbb{P}\left(s_{k}=\infty\right)=1 \Rightarrow$ $\mathbb{P}(s=\infty)=1$ by definition $s=\lim _{k \rightarrow \infty} s_{k}$. The proof of Theorem 3.1 is completed.

\section{Appendix B. Proof of Theorem 3.2}

We only need to prove item 1) of this theorem because proof of item 2) and item 3 is just an application of that of item 1). Actually, since $M(\boldsymbol{X})=\alpha_{3}\left(\|\boldsymbol{X}\|_{\mathcal{H}}\right)$ is strictly positive if $\boldsymbol{X} \neq 0$ we have $\mathbb{P}\left\{\lim _{t \rightarrow \infty}\|\boldsymbol{X}(t)\|_{\mathcal{H}}=0\right\}=1$ for all $\boldsymbol{X}_{0} \in \mathcal{H}$. This together with a.s. global stability proved in item 1) implies a.s. sure global asymptotic stability of the equilibrium. Moreover, since $M(\boldsymbol{X})=\alpha_{3}(\|\boldsymbol{X}\|)$, which is a class $\mathcal{K}_{\infty}$ function, is strictly positive if $\boldsymbol{X} \neq 0$ and radially unbounded in $\|\boldsymbol{X}\|_{\mathcal{H}}$, we have $\mathbb{P}\left\{\lim _{t \rightarrow \infty}\|\boldsymbol{X}(t)\|_{\mathcal{H}} \leq \alpha_{3}^{-1}\left(\varepsilon_{0}\right)\right\}=1$ for all $\boldsymbol{X}_{0} \in \mathcal{H}$. This together with a.s. sure global stability proved in item 1$)$ implies a.s. global practical stability of the equilibrium.

We now prove item 1). Since all the conditions of Theorem 3.1 hold for any $\boldsymbol{X}_{0} \in \mathcal{H}$, the system (16) has a unique variational solution for all $t \in\left[t_{0}, \infty\right)$. Since applying a stopping time procedure and a bounded convergence theorem, see Mao (2007), shows that the solution to (16) is also a strong Markov process under conditions specified in Theorem 3.1, it is sufficient to consider the initial data $\boldsymbol{X}_{0}$ to be deterministic.

\section{Appendix B.1. Almost sure global stability}

We first show that the system (16) is almost sure globally stable. The technique in Deng et al. (2001) is used at places in our proof. Applying the Itô formula to $U(\boldsymbol{X}, t)$ and the solution $\boldsymbol{X}(t)$ of (16) gives

$$
\begin{aligned}
U(\boldsymbol{X}(t), t) & =U(\boldsymbol{X}(s), s)+\int_{s}^{t} \mathcal{L} U(\boldsymbol{X}(r), r) d r+\int_{s}^{t}\left\langle U_{\boldsymbol{X}}(\boldsymbol{X}(r), r), \boldsymbol{G}(\boldsymbol{X}(r), r) d \boldsymbol{W}(r)\right\rangle_{\mathcal{H}} \\
& \leq U(\boldsymbol{X}(s), s)+\int_{s}^{t}\left\langle U_{\boldsymbol{X}}(\boldsymbol{X}(r), r), \boldsymbol{G}(\boldsymbol{X}(r), r) d \boldsymbol{W}(r)\right\rangle_{\mathcal{H}},
\end{aligned}
$$

where we have used the condition (30). The inequality in (B.1) implies that $U(\boldsymbol{X}(t), t)$ is a supermartingale with respect to the filtration $\left\{\mathcal{G}_{t}\right\}$ generated by $\boldsymbol{W}(\cdot)$. By the supermartingale inequality Mao (2007), for any function $\delta(\cdot) \in \mathcal{K}_{\infty}$, we have

$$
\mathbb{P}\left\{\sup _{t_{0} \leq s \leq t} U(\boldsymbol{X}(s), s) \geq \delta\left(U\left(\boldsymbol{X}_{0}, t_{0}\right)\right)\right\} \leq \frac{U\left(\boldsymbol{X}_{0}, t_{0}\right)}{\delta\left(U\left(\boldsymbol{X}_{0}, t_{0}\right)\right)}
$$

for all $t \in\left[t_{0}, \infty\right]$ and $\left.U\left(\boldsymbol{X}_{0}, t_{0}\right)\right) \neq 0$. Thus,

$$
\mathbb{P}\left\{\sup _{t_{0} \leq s \leq t} U(\boldsymbol{X}(s), s)<\delta\left(U\left(\boldsymbol{X}_{0}, t_{0}\right)\right)\right\} \geq 1-\frac{U\left(\boldsymbol{X}_{0}, t_{0}\right)}{\delta\left(U\left(\boldsymbol{X}_{0}, t_{0}\right)\right)}
$$

for all $t \in\left[t_{0}, \infty\right)$ and $\left.U\left(\boldsymbol{X}_{0}, t_{0}\right)\right) \neq 0$. Using (29) shows that $\sup _{t_{0} \leq s \leq t} U(\boldsymbol{X}(s), s)<\delta\left(U\left(\boldsymbol{X}_{0}, t_{0}\right)\right)$ implies that $\sup _{t_{0} \leq s \leq t}\|\boldsymbol{X}(s)\|_{\mathcal{H}}<\varrho\left(\left\|\boldsymbol{X}_{0}\right\|_{\mathcal{H}}\right)$, where $\varrho=\alpha_{1}^{-1} \circ \delta \circ \alpha_{2}$. For a given $\varepsilon>0$, we can always choose $\delta \in \mathcal{K}_{\infty}$ such that $\frac{U\left(\boldsymbol{X}_{0}, t_{0}\right)}{\delta\left(U\left(\boldsymbol{X}_{0}, t_{0}\right)\right)} \leq \varepsilon$. Thus, we can write (B.3) as

$$
\mathbb{P}\left\{\sup _{t_{0} \leq s \leq t}\|\boldsymbol{X}(s)\|_{\mathcal{H}}<\varrho\left(\left\|\boldsymbol{X}_{0}\right\|_{\mathcal{H}}\right)\right\} \geq 1-\varepsilon
$$

for all $t \in\left[t_{0}, \infty\right)$ and $\boldsymbol{X}_{0} \neq 0$. This further implies that

$$
\mathbb{P}\left\{\|\boldsymbol{X}(t)\|_{\mathcal{H}}<\varrho\left(\left\|\boldsymbol{X}_{0}\right\|_{\mathcal{H}}\right)\right\} \geq 1-\varepsilon
$$

for all $t \in\left[t_{0}, \infty\right)$ and $\boldsymbol{X}_{0} \in \mathcal{H} \backslash\{0\}$. This proves almost sure global stability of (16). 
Appendix B.2. Almost sure convergence of $\mathbb{P}\{M(X(t))\}$

We first decompose the sample space into three mutually exclusive events:

$$
\begin{aligned}
& \Delta_{1}:=\left\{\varpi: \lim _{t \rightarrow \infty} \sup M(X(t, \varpi))=0\right\}, \\
& \Delta_{2}:=\left\{\varpi: \lim _{t \rightarrow \infty} \inf M(X(t, \varpi))>0\right\}, \\
& \Delta_{3}:=\left\{\varpi: \lim _{t \rightarrow \infty} \inf M(X(t, \varpi))=0 \text { and } \lim _{t \rightarrow \infty} \sup M(X(t, \varpi))>0\right\} .
\end{aligned}
$$

Thus, in order to prove almost sure global stability of (16), we just need to show that $\mathbb{P}\left\{\Delta_{2}\right\}=0$ and $\mathbb{P}\left\{\Delta_{3}\right\}=0$. Applying the Itô formula to $U(\boldsymbol{X}, t)$ and the solution $\boldsymbol{X}(t)$ of (16) gives

$$
U(\boldsymbol{X}(t), t)=U\left(\boldsymbol{X}_{0}, t_{0}\right)+\int_{t_{0}}^{t} \mathcal{L} U(\boldsymbol{X}(r), r) d r+\int_{t_{0}}^{t}\left\langle U_{\boldsymbol{X}}(\boldsymbol{X}(r), r), \boldsymbol{G}(\boldsymbol{X}(r), r) d \boldsymbol{W}(r)\right\rangle_{\mathcal{H}} .
$$

Since $\int_{t_{0}}^{t}\left\langle U_{\boldsymbol{X}}(\boldsymbol{X}(r), r), \boldsymbol{G}(\boldsymbol{X}(r), r) d \boldsymbol{W}(r)\right\rangle_{\mathcal{H}}$ is a martingale, we have

$$
\mathbb{E}\left\{\int_{t_{0}}^{t}\left\langle U_{\boldsymbol{X}}(\boldsymbol{X}(r), r), \boldsymbol{G}(\boldsymbol{X}(r), r) d \boldsymbol{W}(r)\right\rangle_{\mathcal{H}}\right\}=0 .
$$

By taking expectancy both sides of (B.7) and using (30) and (B.8), we have

$$
\begin{aligned}
& \mathbb{E}\{U(\boldsymbol{X}(t), t)\} \leq U\left(\boldsymbol{X}_{0}, t_{0}\right)-\mathbb{E}\left\{\int_{t_{0}}^{t} M(\boldsymbol{X}(r)) d r\right\} \\
\Rightarrow & \mathbb{E}\left\{\int_{t_{0}}^{t} M(\boldsymbol{X}(r)) d r\right\} \leq U\left(\boldsymbol{X}_{0}, t_{0}\right) \\
\Rightarrow & \int_{t_{0}}^{\infty} M(\boldsymbol{X}(r)) d r \leq \infty, \quad \text { a.s. }
\end{aligned}
$$

Thus, $\lim _{t \rightarrow \infty} \inf M(X(t))=0$ a.s. That is

$$
\mathbb{P}\left\{\Delta_{2}\right\}=0 .
$$

We now prove $\mathbb{P}\left\{\Delta_{3}\right\}=0$ by contradiction. Suppose that $\mathbb{P}\left\{\Delta_{3}\right\} \neq 0$. Then there exist $\mu_{0}>0$ and $\mu_{1}>0$ such that

$$
\mathbb{P}\left\{M(X(t)) \text { crosses from below } \mu_{1} \text { to above } 2 \mu_{1} \text { and back infinitely many times }\right\} \geq \mu_{0} .
$$

Since the initial data $\boldsymbol{X}_{0}$ is bounded, we can find a nonnegative constant $h$ such that $\left\|\boldsymbol{X}_{0}\right\|_{\mathcal{H}}<h$ and define a stopping time $s_{h}$ by

$$
s_{h}:=\inf \left\{t \geq t_{0}:\|\boldsymbol{X}(t)\|_{\mathcal{H}}>h\right\} .
$$

Then the local conditions of Theorem 3.2 imply that there exists a constant $k_{h}>0$ such that

$$
\|\boldsymbol{F}(\boldsymbol{X}(s), s)\|_{\mathcal{H}}^{2}+\|\boldsymbol{G}(\boldsymbol{X}(s), s)\|_{\mathscr{L}_{2}^{0}}^{2} \leq k_{h},
$$

for any $t_{0} \leq s \leq s_{h}$. Now for any $t \geq t_{0}$, the Itô formula gives

$$
\begin{aligned}
\left\|\boldsymbol{X}(t)-\boldsymbol{X}_{0}\right\|_{\mathcal{H}}^{2} & =\int_{t_{0}}^{t} \mathcal{L}\left\|\boldsymbol{X}(t)-\boldsymbol{X}_{0}\right\|_{\mathcal{H}}^{2}+\int_{t_{0}}^{t}\left\langle\left(\left\|\boldsymbol{X}(t)-\boldsymbol{X}_{0}\right\|_{\mathcal{H}}^{2}\right)_{\boldsymbol{X}}, \boldsymbol{G}(\boldsymbol{X}(r), r) d \boldsymbol{W}(r)\right\rangle_{\mathcal{H}} \\
& =2 \int_{t_{0}}^{t}\left\langle\boldsymbol{X}(r)-\boldsymbol{X}_{0}, \boldsymbol{F}(\boldsymbol{X}(r), r)\right\rangle_{\mathcal{H}} d r+\int_{t_{0}}^{t}\|\boldsymbol{G}(\boldsymbol{X}(r), r)\|_{\mathscr{L}_{0}^{2}}^{2} d r+2 \int_{t_{0}}^{t}\left\langle\left(\boldsymbol{X}(t)-\boldsymbol{X}_{0}\right), \boldsymbol{G}(\boldsymbol{X}(r), r) d \boldsymbol{W}(r)\right\rangle_{\mathcal{H}} .
\end{aligned}
$$

Thus, we have

$$
\begin{aligned}
\mathbb{E}\left\{\sup _{t_{0} \leq s \leq t}\left\|\boldsymbol{X}\left(s \wedge s_{h}\right)-\boldsymbol{X}_{0}\right\|_{\mathcal{H}}^{2}\right\} & \leq 2 \mathbb{E}\left\{\sup _{t_{0} \leq s \leq t} \int_{t_{0}}^{s \wedge s_{h}}\left|\left\langle\boldsymbol{X}(r)-\boldsymbol{X}_{0}, \boldsymbol{F}(\boldsymbol{X}(r), r)\right\rangle_{\mathcal{H}}\right| d r\right\}+\mathbb{E}\left\{\sup _{t_{0} \leq s \leq t} \int_{t_{0}}^{s \wedge s_{h}}\|\boldsymbol{G}(\boldsymbol{X}(r), r)\|_{\mathscr{L}_{0}^{2}}^{2} d r\right\} \\
& \left.+2 \mathbb{E}\left\{\sup _{t_{0} \leq s \leq t} \int_{t_{0}}^{s \wedge s_{h}} \mid\left\langle\left(\boldsymbol{X}(t)-\boldsymbol{X}_{0}\right), \boldsymbol{G}(\boldsymbol{X}(r), r) d \boldsymbol{W}(r)\right\rangle_{\mathcal{H}}\right\}\right\} .
\end{aligned}
$$

Using (B.13), we have

$$
\mathbb{E}\left\{\sup _{t_{0} \leq s \leq t} \int_{t_{0}}^{s \wedge s_{h}}\left|\left\langle\boldsymbol{X}(r)-\boldsymbol{X}_{0}, \boldsymbol{F}(\boldsymbol{X}(r), r)\right\rangle_{\mathcal{H}}\right| d r\right\}+\mathbb{E}\left\{\sup _{t_{0} \leq s \leq t} \int_{t_{0}}^{s \wedge s_{h}}\|\boldsymbol{G}(\boldsymbol{X}(r), r)\|_{\mathscr{L}_{0}^{2}}^{2} d r\right\} \leq\left(h^{2}+\left\|\boldsymbol{X}_{0}\right\|_{\mathcal{H}}^{2}+k_{h}\right)\left(t-t_{0}\right) .
$$


Next, to compute the upper-bound of the last term in the right hand side of (B.15), we use the Burkholder-DavisGundy inequality, see Liu (2006), to obtain

$$
\begin{aligned}
2 \mathbb{E}\left\{\sup _{t_{0} \leq s \leq t} \int_{t_{0}}^{s \wedge s_{h}}\left|\left\langle\left(\boldsymbol{X}(t)-\boldsymbol{X}_{0}\right), \boldsymbol{G}(\boldsymbol{X}(r), r) d \boldsymbol{W}(r)\right\rangle_{\mathcal{H}}\right|\right\} & \leq c_{0} \mathbb{E}\left\{\left[\int_{t_{0}}^{t \wedge s_{h}}\left\|\boldsymbol{X}(t)-\boldsymbol{X}_{0}\right\|_{\mathcal{H}}^{2}\|\boldsymbol{G}(\boldsymbol{X}(r), r)\|_{\mathscr{L}_{0}^{2}} d r\right]^{\frac{1}{2}}\right\} \\
& \leq \frac{1}{4} \mathbb{E}\left\{\sup _{t_{0} \leq s \leq t}\left\|\boldsymbol{X}\left(s \wedge s_{h}\right)-\boldsymbol{X}_{0}\right\|_{\mathcal{H}}^{2}\right\}+c_{0}^{2} \mathbb{E}\left\{\int_{t_{0}}^{t \wedge s_{h}}\|\boldsymbol{G}(\boldsymbol{X}(r), r)\|_{\mathscr{L}_{0}^{2}}^{2} d r\right\} \\
& \leq \frac{1}{4} \mathbb{E}\left\{\sup _{t_{0} \leq s \leq t}\left\|\boldsymbol{X}\left(s \wedge s_{h}\right)-\boldsymbol{X}_{0}\right\|_{\mathcal{H}}^{2}\right\}+c_{0}^{2} k_{h}\left(t-t_{0}\right),
\end{aligned}
$$

where $c_{0}$ is a positive constant. Now, substituting (B.16) and (B.17) into (B.15) yields

$$
\frac{3}{4} \mathbb{E}\left\{\sup _{t_{0} \leq s \leq t}\left\|\boldsymbol{X}\left(s \wedge s_{h}\right)-\boldsymbol{X}_{0}\right\|_{\mathcal{H}}^{2}\right\} \leq c_{1}\left(t-t_{0}\right)
$$

where $c_{1}=h^{2}+\left\|\boldsymbol{X}_{0}\right\|_{H}^{2}+k_{h}+c_{0}^{2} k_{h}$. The above inequality together with Chebyshev's inequality, for any $\eta>0$, gives

$$
\mathbb{P}\left\{\sup _{t_{0} \leq s \leq t}\left\|\boldsymbol{X}\left(s \wedge s_{h}\right)-\boldsymbol{X}_{0}\right\|_{\mathcal{H}}^{2}>\eta\right\} \leq \frac{c_{1}}{\eta^{2}}\left(t-t_{0}\right) .
$$

Since $M$ is continuous in $\mathcal{H}$, it must be uniformly continuous in the closed ball $B_{h}:=\left\{\boldsymbol{X} \in \mathcal{H}:\|\boldsymbol{X}\|_{\mathcal{H}} \leq \varrho(h)\right\}$ with $\varrho=\alpha_{1}^{-1} \circ \delta \circ \alpha_{2}$. Thus, for a given $\mu>0$ we can always choose a function $\gamma \in \mathcal{K}$ such that $|M(\boldsymbol{X})-M(\boldsymbol{Y})| \leq \mu$ for all $\boldsymbol{X}, \boldsymbol{Y} \in B_{h}$ and $t \in\left[t_{0}, \infty\right)$. So, for any $\mu_{2}>0$ from (B.5) and (B.19) we have

$$
\begin{aligned}
\mathbb{P}\left\{\sup _{t_{0} \leq s \leq t} \mid M(\boldsymbol{X}(s))-\right. & \left.M\left(\boldsymbol{X}_{0}\right) \mid>\mu_{2}\right\}=\mathbb{P}\left\{\sup _{t_{0} \leq s \leq t}\left|M(\boldsymbol{X}(s))-M\left(\boldsymbol{X}_{0}\right)\right|>\mu_{2}, \sup _{t_{0} \leq s \leq t}\|\boldsymbol{X}(s)\|_{\mathcal{H}}<\varrho(h)\right\} \\
& +\mathbb{P}\left\{\sup _{t_{0} \leq s \leq t}\left|M(\boldsymbol{X}(s))-M\left(\boldsymbol{X}_{0}\right)\right|>\mu_{2}, \sup _{t_{0} \leq s \leq t}\|\boldsymbol{X}(s)\|_{\mathcal{H}} \geq \varrho(h)\right\} \\
\leq & \mathbb{P}\left\{\sup _{t_{0} \leq s \leq t}\left\|\boldsymbol{X}(s)-\boldsymbol{X}_{0}\right\|_{\mathcal{H}}>\gamma\left(\mu_{2}\right), \sup _{t_{0} \leq s \leq t}\|\boldsymbol{X}(s)\|_{\mathcal{H}}<\varrho(h)\right\}+\mathbb{P}\left\{\sup _{t_{0} \leq s \leq t}\|\boldsymbol{X}(s)\|_{\mathcal{H}} \geq \varrho(h)\right\} \\
\leq & \mathbb{P}\left\{\sup _{t_{0} \leq s \leq t}\left\|\boldsymbol{X}\left(s \wedge s_{\varrho(h)}\right)-\boldsymbol{X}_{0}\right\|_{\mathcal{H}}>\gamma\left(\mu_{2}\right)+\mathbb{P}\left\{\sup _{t_{0} \leq s \leq t}\|\boldsymbol{X}(s)\|_{\mathcal{H}} \geq \varrho(h)\right\} \leq \frac{c_{1}\left(t-t_{0}\right)}{\gamma^{2}\left(\mu_{2}\right)}+\mu .\right.
\end{aligned}
$$

Picking $\mu=\frac{1}{2}$, for any $\mu_{2}>0, \exists t^{\diamond}=t^{\diamond}\left(h, \mu_{2}\right)$ such that

$$
\mathbb{P}\left\{\sup _{t_{0} \leq s \leq t^{\circ}}\left|M(X(s))-M\left(X_{0}\right)\right| \leq \mu_{2}\right\} \geq \frac{1}{4} .
$$

Now, let $\mu_{1}>0$ be a constant to be picked and us define a sequence of stopping times

$$
\begin{aligned}
& \sigma_{1}:=\inf \left\{t \geq t_{0}: M(X(t))<\mu_{1}\right\}, \\
& \sigma_{2 k}:=\inf \left\{t \geq \sigma_{2 k-1}: M(X(t))>2 \mu_{1}\right\}, k=1,2, \ldots, \\
& \sigma_{2 k+1}:=\inf \left\{t \geq \sigma_{2 k}: M(X(t))<\mu_{1}\right\}, k=1,2, \ldots,
\end{aligned}
$$

where $\inf \emptyset:=\infty$. From (B.9), we have

$$
\begin{aligned}
\infty & >\mathbb{E}\left\{\int_{t_{0}}^{\infty} M(\boldsymbol{X}(r)) d r\right\} \\
& \geq \sum_{k=1}^{\infty} \mathbb{E}\left\{\mathcal{J}_{\left\{\sigma_{2 k}<s_{h}\right\}} \int_{\sigma_{2 k}}^{\sigma_{2 k+1}} M(\boldsymbol{X}(r)) d r\right\} \\
& \geq \mu_{1} \sum_{k=1}^{\infty} \mathbb{E}\left\{\mathcal{J}_{\left\{\sigma_{2 k}<s_{h}\right\}}\left(\sigma_{2 k}-\sigma_{2 k+1}\right)\right\} \\
& =\mu_{1} \sum_{k=1}^{\infty} \mathbb{E}\left\{\mathcal{J}_{\left\{\sigma_{2 k}<s_{h}\right\}} \mathbb{E}\left\{\sigma_{2 k}-\sigma_{2 k+1} \mid \mathcal{F}_{\sigma_{2 k}}\right\}\right\} .
\end{aligned}
$$

Now, by the strong Markov property of solutions of (16), on $\left\{\sigma_{2 k}<s_{h}\right\}$, the vector $\tilde{\boldsymbol{X}}(\cdot):=\boldsymbol{X}\left(\cdot+\sigma_{2 k}\right)$ under the conditional distribution $\mathbb{P}\left(\cdot \mid \mathcal{F}_{\sigma_{2 k}}\right)$ is the same as that of a solution of (16) with $t$ being replaced by $t+\sigma_{2 k}$ and the 
initial data satisfying $\tilde{\boldsymbol{X}}_{0}<h$. Thus, the estimate (B.21) applies with $\tilde{\boldsymbol{X}}(\cdot)$ in place of $\boldsymbol{X}(\cdot)$ and $\mathbb{P}\left\{\cdot \mid \mathcal{F}_{\sigma_{2 k}}\right\}$ in place of $\mathbb{P}\{\cdot\}$ on $\left\{\sigma_{2 k}<s_{h}\right\}$. Picking $\mu_{1}=2 \mu_{2}$ results in

$$
\begin{aligned}
\mathbb{E}\left\{\sigma_{2 k}-\sigma_{2 k+1} \mid \mathcal{F}_{\sigma_{2 k}}\right\} & \geq \mathbb{E}\left\{\left(\sigma_{2 k}-\sigma_{2 k+1}\right) \mathcal{J}_{\sup _{t_{0} \leq \leq \leq t^{\circ}}\left|M(\tilde{\boldsymbol{X}}(t))-M\left(\tilde{\boldsymbol{X}}\left(t_{0}\right)\right)\right| \leq \frac{\mu_{1}}{2}} \mid \mathcal{F}_{\sigma_{2 k}}\right\} \\
& \geq t^{\diamond} \mathbb{P}\left\{\sup _{t_{0} \leq t \leq t^{\circ}}\left|M(\tilde{\boldsymbol{X}}(t))-M\left(\tilde{\boldsymbol{X}}\left(t_{0}\right)\right)\right| \leq \frac{\mu_{1}}{2} \mid \mathcal{F}_{\sigma_{2 k}}\right\} \geq \frac{t^{\diamond}}{4}
\end{aligned}
$$

on $\left\{\sigma_{2 k}<s_{h}\right\}$, where $t^{\diamond}=t^{\diamond}\left(h, \frac{\mu_{1}}{2}\right)$ and $\tilde{\boldsymbol{X}}(\cdot)=\boldsymbol{X}\left(\cdot+\sigma_{2 k}\right)$. Substituting (B.24) into (B.23) yields

$$
\frac{t^{\diamond} \mu_{1}}{4} \sum_{k=1}^{\infty} \mathbb{P}\left\{\sigma_{2 k}<s_{h}\right\}<\infty .
$$

This follows from the Borel-Canelli lemma that

$$
\mathbb{P}\left\{\sigma_{2 k}<s_{h} \text { for infinitely many } k\right\}=0 .
$$

Since

$$
\begin{aligned}
\left\{\sigma_{2 k}<s_{h} \text { for infinitely many } k\right\}= & \left\{\sigma_{2 k}<s_{h} \text { for infinitely many } k \text { and } s_{h}=\infty\right\} \\
& \cup\left\{\sigma_{2 k}<s_{h} \text { for infinitely many } k \text { and } s_{h}<\infty\right\} .
\end{aligned}
$$

Thus,

$$
\mathbb{P}\left\{\sigma_{2 k}<s_{h} \text { for infinitely many } k \text { and } s_{h}=\infty\right\}=0 .
$$

Now, from (29) we have

$$
\mathbb{P}\left\{s_{h}=\infty\right\} \geq \mathbb{P}\left\{\sup _{t \geq t_{0}}\|\boldsymbol{X}(t)\|_{\mathcal{H}}<h\right\} \geq \mathbb{P}\left\{\sup _{t \geq t_{0}} U(\boldsymbol{X}(t), t)<\alpha_{1}(h)\right\} \geq 1-\frac{U\left(\boldsymbol{X}_{0}, t_{0}\right)}{\alpha_{1}(h)} .
$$

Letting $h \rightarrow \infty$ yields $\mathbb{P}\left\{s_{h}=\infty\right\}=1$, which implies that $\mathbb{P}\left\{\sigma_{2 k}<\infty\right.$ for infinitely many $\left.k\right\}=0$. This contradicts (B.11). Thus, we must have $\mathbb{P}\left\{\Delta_{3}\right\}=0$. Proof of Theorem 3.2 is completed.

\section{Appendix C. Proof of Theorem 4.1}

\section{Appendix C.1. Proof of existence and uniqueness}

To prove existence and uniqueness of the variational solution of the closed-loop system consisting of (35), (36), and (49), we apply Theorem 3.1. Thus, we just need to verify that the closed-loop system satisfies all conditions of Theorem 3.1.

\section{Appendix C.1.1. Verifying Assumption 3.1}

The continuity condition holds as seen from the expression of $A_{1 L}(t)$ and $A_{2 L}(t)$. To verify the second item of Assumption 3.1, we have the linear system of the closed-loop system consisting of (35), (36), and (49) as

$$
\begin{aligned}
& d X_{1}=X_{2} d t, \\
& d X_{2}=\left[A_{1 L}(t) X_{1}+A_{2 L}(t) X_{2}+f_{0}(t)\right] d t+\boldsymbol{g}_{0}^{T}(t) d \boldsymbol{W}_{0}(t), \\
& X_{1}\left(t_{0}\right):=X_{10}=u_{0}, X_{2}\left(t_{0}\right):=X_{20}=u_{1},
\end{aligned}
$$

subject to the boundary conditions

$$
\begin{aligned}
& X_{1}^{B 0}=0, \mathbb{D}^{2} X_{1}^{B 0}=0, \mathbb{D}^{2} X_{1}^{B L}=0, \\
& A_{B L}(t) X_{1}^{B L}+k_{B}\left(\gamma X_{1}^{B L}+X_{2}^{B L}\right)=0,
\end{aligned}
$$

where $A_{1 L}(t), A_{2 L}(t)$, and $A_{B L}(t)$ are defined in (34), $g_{0}(t)=\operatorname{col}\left(g_{5}(t), g_{6}(t), g_{7}(t)\right)$ with $g_{i}(t)$ given in (9), and $W_{0}(t)=\operatorname{col}\left(W_{5}(t), W_{6}(t), W_{7}(t)\right)$. Let us define

$$
\hat{U}_{L}(\boldsymbol{X}, t)=\frac{\varrho a}{2}\left\|X_{2}\right\|_{H}^{2}+\frac{P_{0}}{2}\left\|\mathbb{D} X_{1}\right\|_{H}^{2}+\frac{E I}{2}\left\|\mathbb{D}^{2} X_{1}\right\|_{H}^{2}+\gamma \varrho a\left\langle X_{1}, X_{2}\right\rangle_{H},
$$


where $\gamma$ is a positive constant specified in Assumption 2.1. It is clear that

$$
\begin{aligned}
& \hat{U}_{L} \geq \frac{\varrho a}{2}(1-\gamma)\left\|X_{2}^{2}\right\|_{H}^{2}+\left(\frac{P_{0}}{2}-2 \gamma \varrho a L^{2}\right)\left\|\mathbb{D} X_{1}\right\|_{H}^{2}+\frac{E I}{2}\left\|\mathbb{D}^{2} X_{1}\right\|_{H}^{2} \geq \epsilon_{1}\left(\left\|X_{1}\right\|_{V}^{2}+\left\|X_{2}\right\|_{H}^{2}\right), \\
& \hat{U}_{L} \leq \frac{\varrho a}{2}(1+\gamma)\left\|X_{2}^{2}\right\|_{H}^{2}+\left(\frac{P_{0}}{2}+2 \gamma \varrho a L^{2}\right)\left\|\mathbb{D} X_{1}\right\|_{H}^{2}+\frac{E I}{2}\left\|\mathbb{D}^{2} X_{1}\right\|_{H}^{2} \leq \epsilon_{2}\left(\left\|X_{1}\right\|_{V}^{2}+\left\|X_{2}\right\|_{H}^{2}\right),
\end{aligned}
$$

where $\epsilon_{1}$ and $\epsilon_{2}$ are some positive constants.

Applying the Itô formula to $\hat{U}_{L}$ along the solution of (C.1) gives

$$
\hat{U}_{L}(\boldsymbol{X}, t)=\hat{U}_{L}\left(\boldsymbol{X}_{0}, t_{0}\right)+\int_{t_{0}}^{t} \mathcal{L} \hat{U}_{L}(\boldsymbol{X}(s), s) d s+\int_{t_{0}}^{t}\left\langle X_{2}(s), \boldsymbol{g}_{0}^{T}(t) d \boldsymbol{W}_{0}(s)\right\rangle_{H},
$$

where

$$
\begin{aligned}
\mathcal{L} \hat{U}_{L} & =\left\langle X_{2}, A_{1 L}(t) X_{1}+A_{2 L}(t) X_{2}+f_{0}(t)\right\rangle_{H}+P_{0}\left\langle\mathbb{D} X_{1}, \mathbb{D} X_{2}\right\rangle_{H}+E I\left\langle\mathbb{D}^{2} X_{1}, \mathbb{D}^{2} X_{2}\right\rangle_{H}+\gamma \varrho a\left\|X_{2}\right\|_{H}^{2}+\gamma\left\langle X_{1}, A_{1 L}(t) X_{1}\right. \\
& \left.+A_{2 L}(t) X_{2}+f_{0}(t)\right\rangle_{H}+\frac{1}{2}\left\|\boldsymbol{Q}_{0}^{\frac{1}{2}}(t) \boldsymbol{g}_{0}\right\|_{H}^{2},
\end{aligned}
$$

where $\boldsymbol{Q}_{0}(t)=\operatorname{row}\left(Q_{5}(t), Q_{6}(t), Q_{7}(t)\right)$. Substituting $A_{1 L}(t) X_{1}$ and $A_{2 L}(t) X_{2}$ defined in (34) into (C.6), and using integration by parts result in

$$
\begin{aligned}
\mathcal{L} \hat{U}_{L} & =-\gamma E I\left\|\mathbb{D}^{2} X_{1}\right\|_{H}^{2}-\gamma P_{0}\left\|\mathbb{D} X_{1}\right\|_{H}^{2}+\gamma \varrho a\left\|X_{2}\right\|_{H}^{2}-\gamma\left\|a_{1}^{\frac{1}{2}}(t) X_{1}\right\|_{H}^{2}-\left\|b_{1}^{\frac{1}{2}}(t) X_{2}\right\|_{H}^{2}+T_{L}+\frac{1}{2}\left\|\boldsymbol{Q}_{0}^{\frac{1}{2}}(t) \boldsymbol{g}_{0}\right\|_{H}^{2} \\
& -\varphi_{B}\left(\gamma X_{1}^{B L}+X_{2}^{B L}\right)^{2},
\end{aligned}
$$

where $T_{L}=-\left\langle\left(a_{1}(t)+\gamma b_{1}(t)\right) X_{1}, X_{2}\right\rangle_{H}+\left\langle\gamma X_{1}+X_{2}, f_{0}(t)\right\rangle_{H}$. It is clear from the expression of $T_{L}$ (in comparison with the expression of $T$ defined in (44) that under Assumption 2.1 there exist functions $k_{L 1}(t)$ and $k_{L 2}(t)$ larger than some positive constant (actually a nonnegative constant is sufficient here) a.e. $t \in\left[t_{0}, \infty\right)$, and a positive constant $k_{L 0}$ such that

$$
\begin{aligned}
\mathcal{L} \hat{U}_{L} & \leq-\gamma E I\left\|\mathbb{D}^{2} X_{1}\right\|_{H}^{2}-\gamma P_{0}\left\|\mathbb{D} X_{1}\right\|_{H}^{2}-\left\|k_{L 1}(t) X_{1}\right\|_{H}^{2}-\left\|k_{L 2}(t) X_{2}\right\|_{H}^{2}+\left\|k_{L 0} f_{0}(t)\right\|_{H}^{2}+\frac{1}{2}\left\|\boldsymbol{Q}_{0}^{\frac{1}{2}}(t) \boldsymbol{g}_{0}\right\|_{H}^{2}, \\
& \leq\left\|k_{L 0} f_{0}(t)\right\|_{H}^{2}+\frac{1}{2}\left\|\boldsymbol{Q}_{0}^{\frac{1}{2}}(t) \boldsymbol{g}_{0}(t)\right\|_{H}^{2},
\end{aligned}
$$

Substituting (C.8) into (C.9) and then taking expectation give

$$
\mathbb{E}\left\{\hat{U}_{L}(\boldsymbol{X}, t)\right\}=\mathbb{E}\left\{\hat{U}_{L}\left(\boldsymbol{X}_{0}, t_{0}\right)\right\}+\mathbb{E}\left\{\int_{t_{0}}^{t}\left(\left\|k_{L 0} f_{0}(s)\right\|_{H}^{2}+\frac{1}{2}\left\|\boldsymbol{Q}_{0}^{\frac{1}{2}}(s) \boldsymbol{g}_{0}(s)\right\|_{H}^{2}\right) d s\right\},
$$

which combines with (C.4) to readily yield the second item of Assumption 3.1. This completes verification of Assumption 3.1.

\section{Appendix C.1.2. Verifying Assumption 3.2}

To verify Assumption 3.2, it is sufficient to show for $X_{1}, Y_{1} \in V$ with $\left\|X_{1}\right\|_{V} \vee\left\|Y_{1}\right\|_{V} \leq \varepsilon$, where $\varepsilon$ is a positive constant, that

$$
\begin{aligned}
& \left\|\left(\mathbb{D} X_{1}\right)^{2} \mathbb{D}^{2} X_{1}\right\|_{H}^{2} \leq b_{3}\left\|X_{1}\right\|_{V}^{2}, \\
& \left\|\left(\mathbb{D} X_{1}\right)^{2} \mathbb{D}^{2} X_{1}-\left(\mathbb{D} Y_{1}\right)^{2} \mathbb{D}^{2} Y_{1}\right\|_{H}^{2} \leq b_{4}\left\|X_{1}-Y_{1}\right\|_{V}^{2},
\end{aligned}
$$

for some positive constants $b_{3}$ and $b_{4}$, because of two facts: 1) all the other terms in $F_{N}$ and $G_{N}$, see (37) and (9), are a polynomial of $X_{1} \in V$ and $X_{2} \in H$; and 2) $\left\|f_{0}(t)\right\|_{H},\left\|g_{5}\right\|_{H},\left\|g_{6}\right\|_{H}$ and $\left\|g_{7}\right\|_{H}$ are bounded by some positive constants due to Assumption 2.1. The first inequality of (C.10) holds since $\left\|\mathbb{D}^{2} X_{1}\right\|_{H} \leq\left\|X_{1}\right\|_{V}$. Thus, we just need to verify the second inequality of (C.10). As such, we have

$$
\begin{aligned}
\left\|\left(\mathbb{D} X_{1}\right)^{2} \mathbb{D}^{2} X_{1}-\left(\mathbb{D} Y_{1}\right)^{2} \mathbb{D}^{2} Y_{1}\right\|_{H} & =\left\|\left(\mathbb{D} X_{1}\right)^{2} \mathbb{D}^{2}\left(X_{1}-Y_{1}\right)+\mathbb{D}\left(X_{1}-Y_{1}\right)\left(\mathbb{D} X_{1}+\mathbb{D} Y_{1}\right) \mathbb{D}^{2} Y_{1}\right\|_{H} \\
& \leq\left\|\left(\mathbb{D} X_{1}\right)^{2}\right\|_{H}\left\|\mathbb{D}^{2}\left(X_{1}-Y_{1}\right)\right\|_{H}+\left\|\mathbb{D}\left(X_{1}-Y_{1}\right)\right\|_{H}\left(\left\|\mathbb{D} X_{1}\right\|_{H}+\left\|\mathbb{D} Y_{1}\right\|_{H}\right)\left\|\mathbb{D}^{2} Y_{1}\right\|_{H},
\end{aligned}
$$

which readily implies the second inequality of (C.10). This completes verification of Assumption 3.2. 


\section{Appendix C.2. Convergence analysis}

Since we have already proved existence and uniqueness of the solution of the closed-loop system, we now need to verify Conditions (29) and (33) of Theorem 3.2 to show almost sure global practical stability of the closed-loop system. Condition (29) holds because we already showed from (41) that $\alpha_{1}\left(\|\boldsymbol{X}\|_{\mathcal{H}}\right) \leq U \leq \alpha_{2}\left(\|\boldsymbol{X}\|_{\mathcal{H}}\right)$. Moreover, it is seen from (50) that there exists a class- $\mathcal{K}_{\infty}$ function $\alpha_{3}\left(\|\boldsymbol{X}\|_{\mathcal{H}}\right)$ such that

$$
\mathcal{L} U \leq-\alpha_{3}\left(\|X\|_{\mathcal{H}}\right)+\sup _{t \geq t_{0}} k_{0}(t)
$$

where we have used $\left\|X_{1}^{2}\right\|_{H} \leq C\left\|X_{1}\right\|_{V}^{\frac{1}{2}}\left\|X_{1}\right\|_{H}^{\frac{7}{2}} \leq C\left\|X_{1}\right\|_{V}^{4}$ with $C$ being a positive constant. Since all the conditions of item 3) of Theorem 3.2 have been verified, the closed-loop system is almost sure global practical stable at the origin. Proof of Theorem 4.1 is completed.

\section{References}

Adams, R.A., Fournier, J.J.F., 2003. Sobolev Spaces. Academic Press, Oxford, UK. 2nd edition.

Anderson, B.D.O., Moore, J.B., 1990. Optimal control: linear quadratic methods. Dover Publications.

Baek, H., Karniadakis, G.E., 2009. Suppressing vortex induced vibrations via passive means. Journal of Fluids and Structures $25,848-866$.

Bohm, M., Krstic, M., Kuchler, S., Sawodny, O., 2014. Modelling and boundary control of a hanging rope immersed in water. Journal of Dynamic Systems, Measurement, and Control 136, 011006-1:011006-15.

Brzezniak, Z., Maslowski, B., Seidler, J., 2005. Stochastic nonlinear beam equations. Probability Theory and Related Fields 132, $119-149$.

Chow, P.L., 2007. Stochastic Partial Differential Equations. Chapman \& Hall/CRC, Boca Raton.

Chow, P.L., Menaldi, J.L., 2014. Stochastic PDE for nonlinear vibration of elastic panels. Differential Integral Equations 12, $419-434$.

Deng, H., Krstic, M., Williams, R., 2001. Stabilization of stochastic nonlinear systems driven by noise of unknown covariance. IEEE Transactions on Automatic Control 46, 1237-1253.

Do, K.D., 2011. Global stabilization of three-dimensional flexible marine risers by boundary control. Ocean Systems Engineering 1, 171-194.

Do, K.D., 2015. Global inverse optimal stabilization of stochastic nonholonomic systems. Systems \& Control Letters 75, 41-55.

Do, K.D., 2016. Boundary control of elastic systems. Journal of Applied Mathematics and Computing 51, 315-339.

Do, K.D., 2017. Boundary control design for extensible marine risers in three dimensional space. Journal of Sound and Vibration 388, $1-19$.

Do, K.D., Pan, J., 2008a. Boundary control of transverse motion of marine risers with actuator dynamics. Journal of Sound and Vibration $318,768-791$

Do, K.D., Pan, J., 2008b. Nonlinear control of an active heave compensation system. Ocean Engineering 35, 558-571.

Do, K.D., Pan, J., 2009. Boundary control of three-dimensional inextensible marine risers. Journal of Sound and Vibration 327, $299-321$.

Dowell, E., 1975. Aeroelasticity of Plates and Shells. Leyden, Noordhoff, The Netherlands.

Elosta, H., Huang, S., Incecik, A., 2013. Dynamic response of steel catenary riser using a seabed interaction under random loads. Ocean Engineering 69, 34-43.

Faltinsen, O., 1993. Sea Loads on Ships and Offshore Structures. Cambridge University Press.

Fard, M.P., Sagatun, S.I., 2001. Exponential stabilization of a transversely vibrating beam by boundary control via lyapunov's direct method. Journal of Dynamic Systems, Measurement, and Control 123, 195-200.

Fung, R.F., Tseng, C.C., 1999. Boundary control of an axially moving string via lyapunov method. Journal of Dynamic Systems, Measurement, and Control 121, 105-110.

Fung, R.F., Wu, J.M., Wu, S.L., 1999. Stabilization of an axially moving string by nonlinear boundary feedback. Journal of Dynamic Systems, Measurement, and Control 121, 117-121.

Gawarecki, L., Mandrekar, V., 2011. Stochastic Differential Equations in Infinite Dimensions with Applications to Stochastic Partial Differential Equations. Springer, Berlin.

Ge, S.S., He, W., How, B., Choo, Y.S., 2010. Boundary control of a coupled nonlinear flexible marine riser. IEEE Transactions on Control systems technology 18, 1080-1091.

He, W., Ge, S.S., How, B.V., Choo, Y.S., Hong, K.S., 2011. Robust adaptive boundary control of a flexible marine riser with vessel dynamics. Automatica 47, 722-732.

Khalil, H., 2002. Nonlinear systems. Prentice Hall.

Khasminskii, R., 1980. Stochastic Stability of Differential Equations. S\&N International, Rockville MD.

Krstic, M., Kanellakopoulos, I., Kokotovic, P., 1995. Nonlinear and adaptive control design. Wiley, New York.

Liu, K., 2006. Stability of infinite dimensional stochastic differential equations with applications. Chapman and Hall/CRC, Boca Raton, FL.

Liu, R., Mandrekar, V., 1997. Stochastic semilinear evolution equations: Lyapunov function, stability and ultimate boundedness. Journal of Mathematical Analysis and Applications 212, 537-553.

Mao, X., 2007. Stochastic Differential Equations and Applications. Woodhead publishing, Cambridge. 2nd edition.

Meirovitch, L., 1997. Principles and Techniques of Vibrations. Prentice-Hall.

Nguyen, T.L., Do, K.D., Pan, J., 2013. Boundary control of two-dimensional marine risers with bending couplings. Journal of Sound and Vibration 332, 3605-3622.

Pardoux, E., 1979. Stochastic partial differential equations and filtering of diffusion processes. Stochastics 3, $127-167$.

Prato, G.D., Zabczyk, J., 1992. Stochastic Equations in Infinite Dimensions. Cambridge University Press, Cambridge, UK.

Richtmyer, R.D., Morton, K.W., 1967. Difference Methods for Initial-Value Problems. John Wiley and Sons, New York. 
Sorensen, A.J., 2005. Structural issues in the design and operation of marine control systems. Annual Reviews in Control 29, $125-149$.

Stanway, R., Burrows, C.R., 1981. Active vibration control of a flexible rotor on flexibly-mounted journal bearings. Journal of Dynamic Systems, Measurement, and Control 103, 383-388.

Torres, L., Verde, C., Vazquez-Hernandez, O., 2015. Parameter identification of marine risers using kalman-like observers. Ocean Engineering 93, 84-97.

Zhang, T., 2007. Large deviations for stochastic nonlinear beam equations. Journal of Functional Analysis 248, $175-201$. 